\title{
Élaboration du rendement du blé d'hiver en conditions de déficit hydrique. I. Étude en lysimètres
}

\author{
P Debaeke *, J Puech, ML Casals \\ avec la collaboration technique de P Petibon \\ Station d'agronomie, Inra, BP 27, F-31326 Castanet-Tolosan cedex, France
}

(Reçu le 30 mai 1995 ; accepté le 23 novembre 1995)

\begin{abstract}
Résumé - Si de nombreux travaux rapportent les effets de défauts d'alimentation hydrique sur le rendement du blé d'hiver et ses composantes, on n'est cependant pas en mesure d'en modéliser les conséquences sur l'indice de récolte. Or cette connaissance est nécessaire au fonctionnement des modèles de simulation qui prédisent le rendement du blé à l'aide de la biomasse aérienne et de l'indice de récolte (IR). C'est pourquoi, à partir d'un dispositif de cases lysimétriques, nous avons cherché à établir les fonctions de réponse du rendement, de la biomasse aérienne et de l'indice de récolte du blé d'hiver à des contraintes hydriques variant par l'intensité, la durée de la période d'application. Les résultats ( 3 années) illustrent la chute de rendement, de biomasse aérienne et de nombre de grains par $\mathrm{m}^{2}$ avec celle du taux de satisfaction des besoins en eau (ETR/ETM), la période gonflement-stade laiteux apparaissant comme la plus sensible au stress hydrique tant pour le rendement que pour l'indice de récolte. Si IR reste stable pour des contraintes modérées, la baisse est rapide en deçà de valeurs d'ETR/ETM de 0,75 jusqu'à une valeur minimale d'IR de 0,19. L'indice de récolte augmente avec la part de l'eau consommée par le blé après l'anthèse et plafonne au-delà de $30 \%$. Un stress hydrique précoce (tallage-début montaison) augmente $\mid R$. À partir de ces résultats, un modèle simplifié de réponse d'IR au déficit hydrique par phase est proposé.
\end{abstract}

blé d'hiver / indice de récolte / contrainte hydrique / lysimètres

Summary - Yield build-up in winter wheat under soil water deficit. I. Lysimeter studies. Numerous papers have reported the effects of water stress on wheat grain yield and its components, but limited information is available for modelling its consequences on harvest index. This knowledge is essential for simulation models which predict crop yield as a result of biomass and harvest index (IR). The biomass and harvest index responses to water-stress treatments differing in their intensity, duration and timing were therefore studied on lysimeters over 3 years. Yield, biomass and grain number decreased with water satisfaction rate (ETR/ETM). The booting-milky grain period was the most sen-

\footnotetext{
* Correspondance et tirés à part.

Rappel des abréviations.

Traitements expérimentaux : A case argilo-limoneuse ; L case limoneuse ; ETM évapotranspiration maximale ; FM floraison-maturité ; FSL floraison-stade laiteux du grain ; GF gonflement-floraison; RG début montaison-gonflement ; RM début montaison-maturité ; SC stress hydrique continu; SF semis-floraison ; SG semis-gonflement ; SLM stade laiteux-maturité ; SR semis-début montaison ; TR tallage-début montaison. Variables : Eff-Gr efficience de l'eau exprimée pour la production de grain (kg par mm); ETM évapotranspiration maximale du blé $(\mathrm{mm})$; ETP évapotranspiration potentielle $(\mathrm{mm})$; ETR évapotranspiration réelle du blé ( $\mathrm{mm})$; IR indice de récolte ; NG nombre de grains $\left(p a r m^{2}\right) ; N_{S} S_{p}$ nombre de jours de stress hydrique de la phase $p ; N_{p}$ nombre de jours de la phase p ; Rdt rendement (grain) ; RFU réserve facilement utilisable ( $\mathrm{mm}$ ).
} 
sitive to water stress. Moderate stress did not affect IR but the decrease was rapid below ETR/ETM values of 0.75 . The minimum value of IR was 0.19. IR increased with the contribution of water consumption after anthesis by up to $30 \%$. Water stress during tillering increased the harvest index. Using these results, a simple model is proposed to predict the phasic response of IR to water stress.

winter wheat / harvest index / water stress / lysimeter

\section{INTRODUCTION}

Dans le sud-ouest de la France, le rendement moyen du blé d'hiver reste bas - voisin de 50 q.ha-1 - et varie fortement selon les années, les unités pédoclimatiques, les systèmes de culture et les types d'exploitation (Maruejouls, 1986). Les contraintes climatiques sont les causes principales de ces rendements faibles et irréguliers (Rellier, 1981) ; ainsi, en région toulousaine, le climat se caractérise par un déficit hydrique plus ou moins marqué et précoce au printemps, aggravé en phase de remplissage du grain par une forte demande climatique (vent desséchant, températures échaudantes, rayonnement élevé...) (Puech et al, 1981).

Face à la sécheresse (sols superficiels, déficit pluviométrique), les adaptations stratégiques et tactiques sont diverses : choix de génotypes esquivant ou tolérant les stress hydrique et thermique (Acevedo, 1991), recours à l'irrigation en période sensible (Deumier, 1987), rationnement précoce de la culture (azote, densité...) (Nolot, 1983), choix du précédent (réserves profondes, structure du sol favorable...) (Marty et Hilaire, 1979). La gestion des interactions entre choix techniques reste cependant délicate à raisonner dans un contexte de déficit hydrique incertain.

C'est pourquoi la recherche d'itinéraires techniques minimisant les risques de sécheresse ou leurs conséquences sur le rendement du blé pourrait tirer parti des modèles de simulation du fonctionnement de la culture et du milieu, qui permettent de gérer des interactions complexes, dans un environnement climatique fluctuant (Fischer, 1985). Plusieurs modèles prédisent le rendement d'une culture de blé soumise à un déficit hydrique, soit en simulant l'élaboration successive des composantes du rendement (nombre d'épis par $\mathrm{m}^{2}$, nombre de grains par épi, poids d'un grain) (Ceres-Wheat, Ritchie et Otter, 1985), soit en simulant la biomasse aérienne et la formation de l'indice de récolte (Epic, Williams et al, 1989). En conditions de stress hydrique marqué, le fonctionnement de ces modèles est souvent décevant (Brisson et Delécolle, 1993). C'est ainsi que, dans le cas d'Epic, il est apparu nécessaire de décomposer les effets sur la biomasse aérienne et l'indice de récolte d'une contrainte hydrique appliquée à différents stades de la culture (Quinones et Cabelguenne, 1990).

Plusieurs auteurs ont étudié les conséquences de contraintes hydriques d'intensité et de durée variables, appliquées à diverses phase du cycle sur le rendement du blé et ses composantes (Fischer, 1973 ; Choudhury et Kumar, 1980 ; Innes et Blackwell, 1981 ; Mogensen et al, 1985). Plus rares sont les travaux qui montrent que l'indice de récolte du blé est affecté par l'intensité et la période d'action du stress hydrique (Donald et Hamblin, 1976 ; Passioura, 1977). C'est pourquoi on ne dispose pas de résultats permettant de modéliser par phase l'effet de contraintes en eau sur l'indice de récolte, option retenue pour l'affinement du modèle Epic. C'est en conditions contrôlées (lysimètres par exemple) que de telles relations peuvent être le plus aisément établies (Day et al, 1978).

L'objectif de ce travail est d'analyser l'effet de contraintes hydriques variant par leur intensité, leur durée et leur période d'action sur le rendement du blé d'hiver et ses composantes, avec un intérêt tout particulier pour la formation de l'indice de récolte, puis de proposer un modèle simple d'effet de la contrainte hydrique sur l'indice de récolte.

\section{MATÉRIEL ET MÉTHODES}

Pour remplir ces deux objectifs, le dispositif lysimétrique de la station d'agronomie de l'Inra situé à Auzeville (Haute-Garonne) a été consacré au blé d'hiver pendant trois années (1982, 1991 et 1994).

\section{Description des lysimètres}

\section{Grandes cases}

Ce dispositif lysimétrique, de type gravimétrique, comprend 16 cases cylindriques de 5-7 $\mathrm{m}^{2}$ variant par leur profondeur $(0,95,1,75$ et $2,25 \mathrm{~m})$ et leur texture. Les cases sont remplies de manière homogène avec un sol limoneux (L) de type boulbène ( $20 \%$ d'argile) ou 
avec un sol argilo-limoneux (A) de type terrefort (34\% d'argile). La réserve utile est de $1,90 \mathrm{~mm} / \mathrm{cm}$ en boulbène et de $1,44 \mathrm{~mm} / \mathrm{cm}$ en terrefort. Le dispositif est soustrait à la pluie par un toit fixe en PVC ; l'arrosage des lysimètres est réalisé à partir d'un système de cadres assurant des apports contrôlés par case. Chacune des cases est munie d'un tube central en aluminium permettant le contrôle des profils hydriques par la méthode neutronique ; le drainage est mesuré en continu.

\section{Petits fûts}

Ce dispositif permanent, non couvert, situé à proximité du précédent, est constitué de 12 cases cylindriques de $0,4 \mathrm{~m}^{2}$ sur une profondeur de $1 \mathrm{~m}$ (Puech et al, 1977). II comprend six types de sol (avec deux répétitions) : S (sable), SL (sable limoneux), LA (limon argileux), Al (argile lourde), ASL (sol Al sur $0-50 \mathrm{~cm}$ puis sol SL sur 50-100 cm), SLA (sol SL sur sol Al). Les réserves utiles de ces sols sont respectivement de $89 \mathrm{~mm}$ (S), $170 \mathrm{~mm}$ (SL), $143 \mathrm{~mm}$ (LA), $132 \mathrm{~mm}$ (Al) et $151 \mathrm{~mm}$ (ASL et SLA).

Quatre évapotranspiromètres non couverts, semés en blé et bien alimentés en eau (pluviométrie + nappe + irrigation), constituent la référence ETM.

\section{Conduite du blé et protocoles d'irrigation}

Les principales techniques culturales appliquées au blé sont présentées au tableau I. Le blé est semé sur l'ensemble des cases et leur environnement. Une protection phytosanitaire d'assurance est pratiquée et l'apport d'azote est ajusté au rendement escompté afin de prévenir le risque de verse.

Les protocoles d'irrigation sont organisés selon trois modalités d'application de la contrainte hydrique (fig 1) : dessèchement (apparition progressive du stress au fur et à mesure de l'épuisement de la réserve), phase (effet d'un stress isolé au cours du cycle), récupération (compensation par le remplissage du grain d'un déficit hydrique avant floraison). La référence ETM est fournie par les évapotranspiromètres en 1982 et par un couple de cases profondes ( $A$ et $L$ ), bien alimentées en eau, en 1991 et 1994.

Le protocole «Dessèchement» a comme objectif l'étude de l'effet d'une contrainte hydrique progressive et d'intensité variable s'installant à la montaison sur l'élaboration du rendement du blé en relation avec le type de sol (texture et profondeur de la case).

En 1982, la conduite est homogène jusqu'au stade 1er nœud ( 9 avril), soit $350 \mathrm{~mm}$ appliqués sur des cases revenues à la capacité au champ, puis deux traitements sont réalisés : i) poursuite de l'irrigation (environ $300 \mathrm{~mm}$ ) sur deux (A et $L$ ) $x$ trois cases $(0,95$, 1,75 et $2,25 \mathrm{~m}$ de profondeur), ii) conduite en sec et mise à contribution des réserves du sol sur les autres cases.

En 1994, les irrigations sont stoppées plus précocement, au stade $1 \mathrm{~cm}$ "épi» (2 mars) après réhumectation quasi complète du profil. La progression du déficit hydrique du sol est suivie sur deux ( $A$ et $L$ ) $x$ trois cases $(0,95,1,75$ et $2,25 \mathrm{~m}$ de profondeur).

Enfin, les petits fûts sont conduits selon la pluviométrie 1981/1982, année à déficit hydrique précoce, soit $450 \mathrm{~mm}$ du semis à la récolte, dont $84 \%$ avant montaison.

Le protocole "Phases" a comme objectif l'étude de l'incidence de stress hydriques d'intensitè variable, appliqués durant différentes phases du cycle, sur l'élaboration du rendement du blé et plus spécialement sur l'indice de récolte.

En 1991, I'alimentation hydrique n'est pas limitante jusqu'au stade trois feuilles, puis des conditions de déficit hydrique sont créées à des phases de développement précises du blé par arrêt anticipé de l'irrigation ; à l'issue de chaque phase, la réhumectation se fait à la capacité au champ, le plus rapidement possible, afin d'assurer une alimentation en eau non limitante par la suite (ETM). Six phases sont étudiées :

- TR : début tallage-début montaison (14 janvier au 20 mars) ;

- RG : début montaison-gonflement (du 20 mars au 29 avril) ;

- GF : gonflement-floraison (29 avril au 28 mai) ;

- FSL : floraison-stade laiteux du grain (28 mai au 10 juin) ;

- SLM : stade laiteux-maturité (10 juin au 5 juillet) ;

- SC : stress continu du début tallage à maturité, avec des apports d'eau de survie (150 $\mathrm{mm}$ au total).

Chaque traitement est répété sur les deux textures (A et $L$ ), ce qui permet de disposer d'une gamme d'intensité de stress hydrique par phase.

En 1994, seules trois phases sont étudiées selon le même protocole mais uniquement en cases argileuses :

Tableau I. Principaux éléments de la conduite culturale du blé en lysimètres.

$\begin{array}{lllrl}\text { Année } & \text { Variété } & \text { Date de semis } & \text { Densité }\left(\mathrm{pl} / \mathrm{m}^{2}\right) & \text { Azote }(\mathrm{kg} / \mathrm{ha}) \\ & & & & - \\ 1981-1982 & \text { Top } & 12 \text { novembre } & 400 & 160 \\ 1990-1991 & \text { Vizir } & 14 \text { novembre } & 300 & 160 \\ 1993-1994 & \text { Soissons } & 17 \text { novembre } & 400 & 125\end{array}$




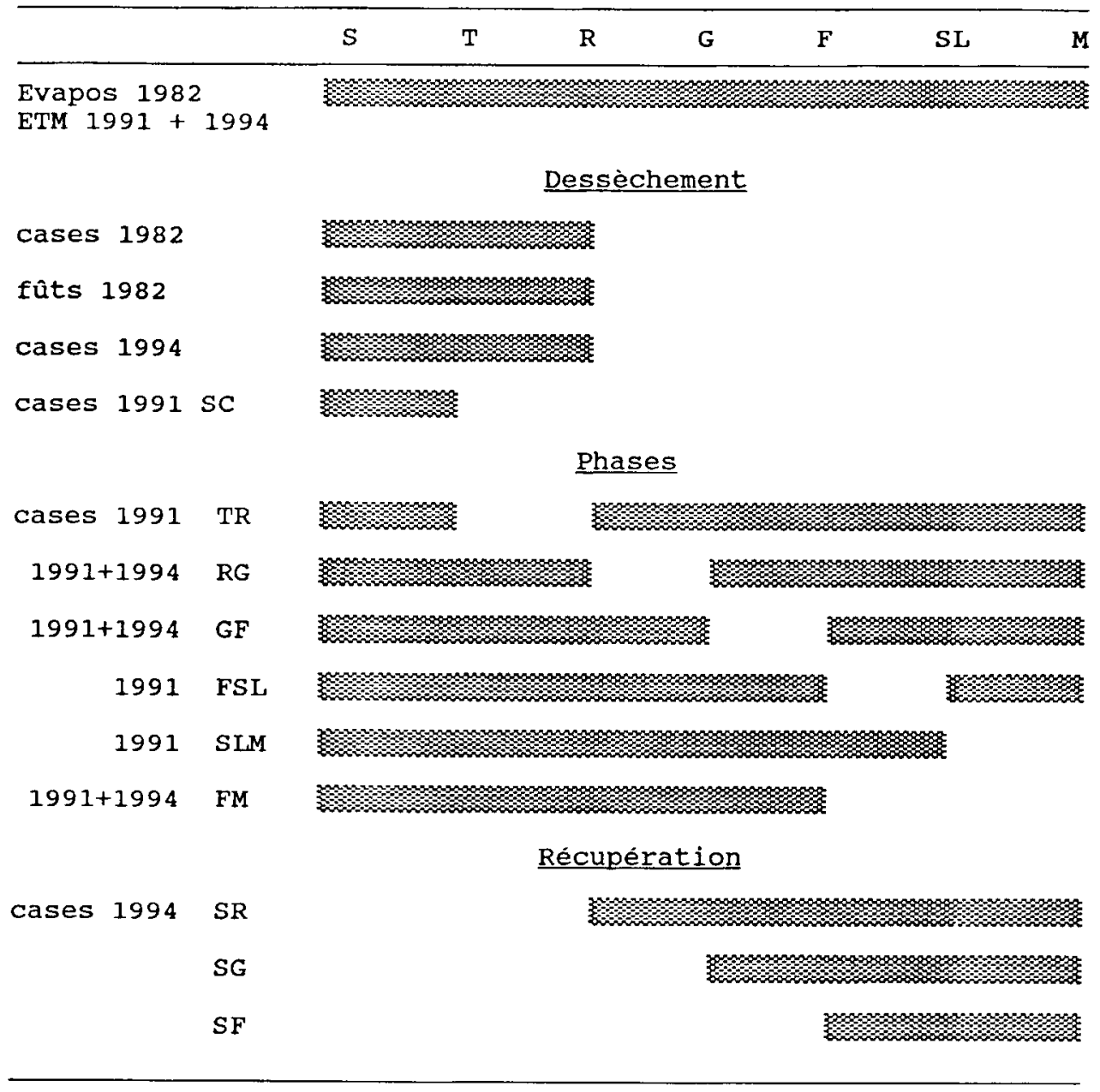

Fig 1. Récapitulatif des traitements expérimentaux. Périodes d'apport d'eau en ombré. S : semis ; $T$ : début de tallage ; $R$ : redressement ; $\mathrm{G}$ : gonflement ; $\mathrm{F}$ : floraison ; SI : stade laiteux ; $M:$ maturité.
- RG : début montaison - gonflement (52 jours) ;

- GF : gonflement - floraison (24 jours) ;

- FM : floraison - maturité (58 jours).

Le protocole «Récupération» a comme objectif l'analyse des possibilités de récupération et de compensation d'un blé ayant subi des périodes de déficit hydrique plus ou moins prolongées avant floraison. En 1994, trois cases argileuses, non réhumectées en profondeur, sont soumises à des périodes de stress continu s'étalant du semis au stade "épi $1 \mathrm{~cm}$ " (SR), soit 84 jours, au gonflement (SG), soit 136 jours ou à la floraison (SF), soit 160 jours.

\section{Échantillonnage et mesures}

Le suivi de l'humidité du sol est réalisé régulièrement par sonde à neutrons. La consommation en eau de chaque case (ETR) est déduite du bilan hydrique.

A maturité, le rendement et ses composantes (nombre d'épis, nombre d'épillets totaux et fertiles (40 à 100 épis selon les années), nombre de grains, poids de 1000 grains, poids spécifique du grain, poids de paille + balles) sont analysés sur $0,5 \mathrm{~m}^{2}$ (1982) et 4 $x 0,3 \mathrm{~m}^{2}$ (1991 et 1994) au centre de la case. Le nombre de plantes par $\mathrm{m}^{2}$ est évalué en sortie d'hiver.
En 1991, des contrôles de croissance (nombre de plantes et de tiges, surface foliaire verte par planimétrie, biomasse aérienne totale et par organe) sont effectués à l'issue de chaque phase sur 4 microplacettes de $0,07 \mathrm{~m}^{2}$.

\section{Analyse statistique}

Les résultats de production sont soumis à une analyse de variance à deux facteurs : texture et phase en 1991, texture et profondeur de sol en 1994. En 1982, un seul facteur est testé, le taux de satisfaction du besoin hydrique (ETR/ETM). Les comparaisons de moyennes se font à l'aide du test de Newman et Keuls au seuil $(p<0,05)$.

\section{Conditions climatiques}

L'année 1982 se caractérise par une sécheresse dès la montaison (ETP journalière : $3,8 \mathrm{~mm}$ ) et par un remplissage en conditions très échaudantes (17 jours où la température maximum dépasse $25^{\circ} \mathrm{C}$, ETP de $5,8 \mathrm{~mm}$ ). L'année 1991 est une année à ETP printanière plus modérée $(3,5 \mathrm{~mm})$ et à conditions de rem- 
plissage plus favorables (8 jours supérieurs à $25^{\circ} \mathrm{C}$, ETP de $5,0 \mathrm{~mm}$ ). L'année 1994 est une année à faible ETP au printemps $(2,8 \mathrm{~mm})$ mais qui présente de fortes températures en fin de cycle (14 jours supérieurs à $25^{\circ} \mathrm{C}$ ). L'ETP reste modérée au cours du remplissage $(4,1 \mathrm{~mm})$.

\section{Intensité et durée de la contrainte hydrique}

Les tableaux II et III présentent les consommations hydriques (ETR) de la levée à la maturité du blé et les indices de stress ETR/ETM. En 1982, par le jeu des profondeurs de sol, des textures et des irrigations, la gamme ETR/ETM est particulièrement large : 0,5 à 0,9 pour les différentes situations de dessèchement du sol. En 1991, à l'exception du traitement SC, on obtient une gamme plus resserrée sur l'ensemble du cycle $(0,6-0,9)$ avec des intensités de stress différentes selon les phases $(0,3-0,9)$. Les stress sont en général moins prononcés en cases limoneuses à plus forte réserve facilement utilisable (RFU). Le traitement
SC permet en revanche de créer un rapport ETR/ETM voisin de 0,3. En 1994, on dispose d'une gamme comparable à celle de 1982, cohérente avec les durées de stress (SR > SG > SF) et les profondeurs de sol $(2,25>1,75>0,95)$.

\section{RÉSULTATS}

\section{Effets d'une contrainte hydrique prolongée (protocole Dessèchement)}

\section{Déficit hydrique à partir de la mi-montaison (cv Top 1982)}

Les nombres d'épis par $\mathrm{m}^{2}$, d'épillets totaux par épi et d'épillets fertiles par épillet ne sont pas affectés significativement par un arrêt de l'irrigation au stade $1^{\mathrm{er}}$ nœud, même si l'on observe

Tableau II. Consommation hydrique et indice de stress (cv Top 1982).

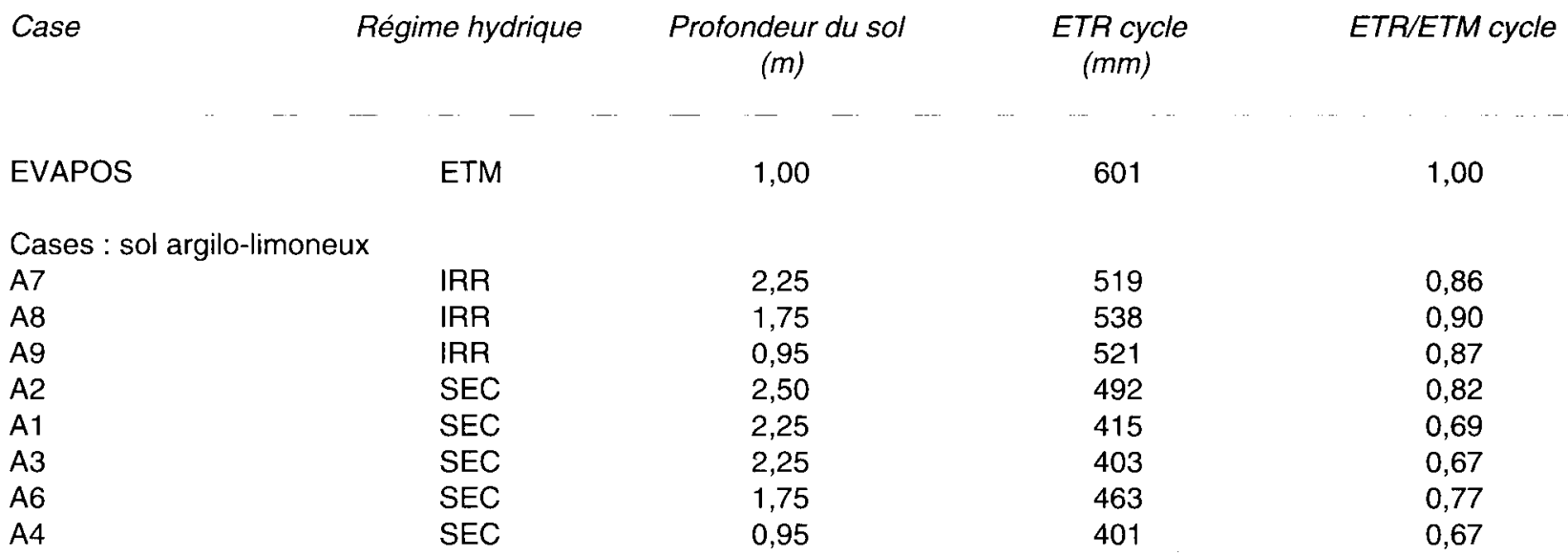

\section{Cases : sol limoneux}

$\begin{array}{lllll}\text { L6 } & \text { IRR } & 2,25 & 408 & 0,68 \\ \text { L7 } & \text { IRR } & 1,75 & 461 & 0,77 \\ \text { L8 } & \text { IRR } & 0,95 & 464 & 0,77 \\ \text { L2 } & \text { SEC } & 2,50 & 445 & 0,74 \\ \text { L1 } & \text { SEC } & 2,25 & 411 & 0,68 \\ \text { L3 } & \text { SEC } & 2,25 & 335 & 0,56 \\ \text { L5 } & \text { SEC } & 1,75 & 307 & 0,51 \\ \text { L4 } & \text { SEC } & 0,95 & 332 & 0,55 \\ \text { Fûts }: \text { diverses textures a } & & & & \\ \text { S } & & & 382 & 0,64 \\ \text { SL } & \text { SEC } & 0,90 & 421 & 0,70 \\ \text { LA } & \text { SEC } & 0,90 & 374 & 0,62 \\ \text { Al } & \text { SEC } & 0,90 & 383 & 0,64 \\ \text { ASL } & \text { SEC } & 0,90 & 434 & 0,72 \\ \text { SLA } & \text { SEC } & 0,90 & 412 & 0,69\end{array}$

a S (sable); SL (sable limoneux) ; LA (limon argileux); Al (argile lourde) ; ASL (sol Al / sol SL), SLA (sol SL / sol Al). 
Tableau III. Consommation hydrique et indice de stress (cV Vizir 1991 et cv Soissons 1994).

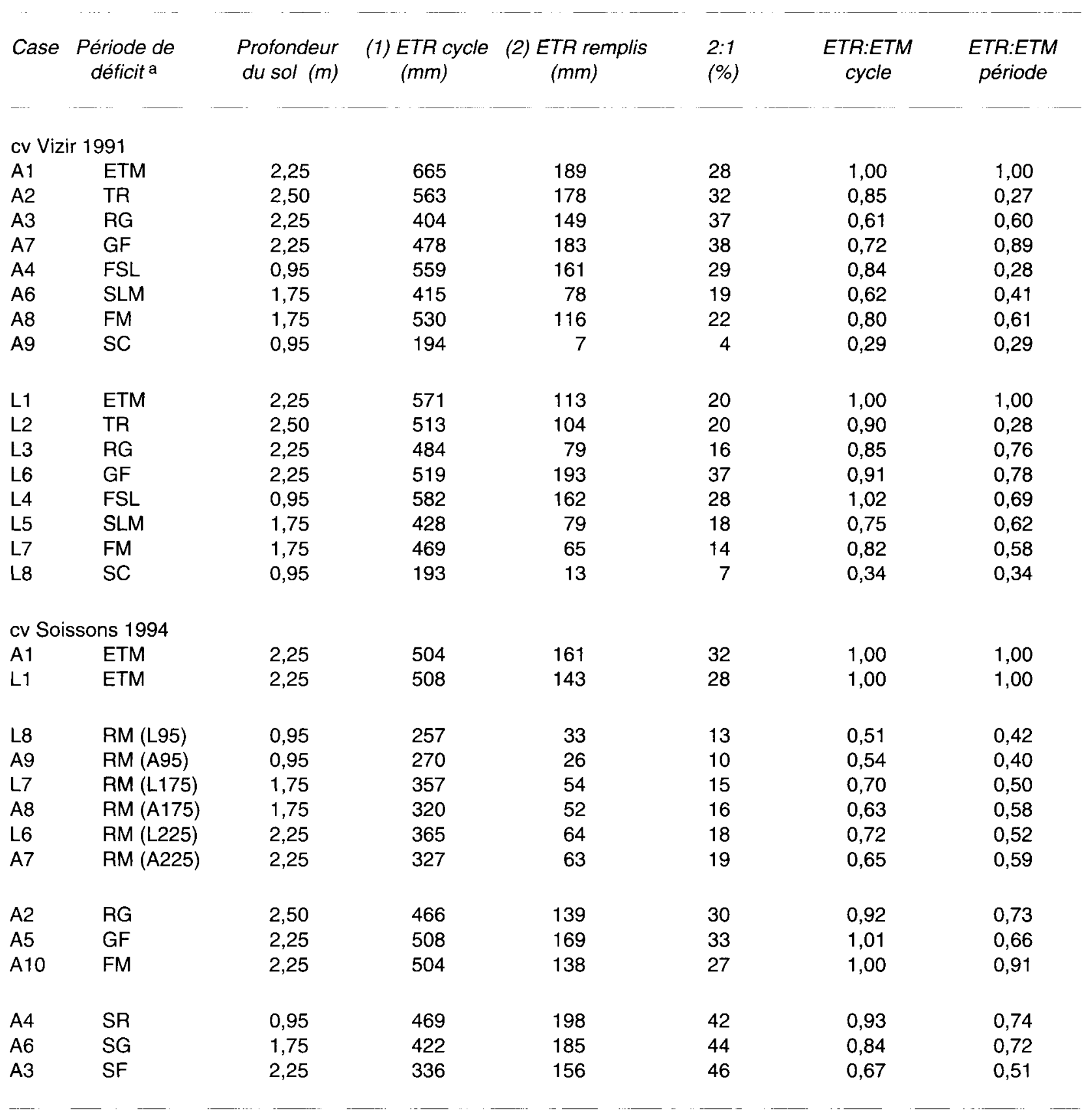

A : sol argilo-limoneux (34\% argile) ; L : sol limoneux (20\% argile); a voir rappel des abréviations.

une tendance à l'augmentation de ces deux dernières composantes avec ETR/ETM (tableau IV). Seule la composante grains par épillet fertile est significativement réduite par la contrainte hydrique extrême ( $-35 \%$ par rapport à I'ETM). En conséquence, le nombre de grains par $\mathrm{m}^{2}$ est sévèrement déprimé ( $-55 \%$ ) par un défaut d'alimentation hydrique de $50 \%$. De même, le poids de 1000 grains augmente avec ETR/ETM, dans les situations où le nombre de grains par $\mathrm{m}^{2}$ n'excède pas 14000 , cette borne correspondant pour la variété Top au début de la concurrence entre grains pour les assimilats (Nolot, 1983). L'augmentation du poids d'un grain ne compense qu'en partie la baisse marquée du nombre de grains pour des contraintes modérées. L'indice de récolte suit la même évolution que le poids d'un grain mais est sévèrement réduit (-29\%) pour la contrainte extrême. L'efficience de l'eau consommée pour la production de grain ou de biomasse ne diffère pas significativement selon ETR/ETM. 
Tableau IV. Effets de l'intensité de la contrainte hydrique (1 ${ }^{\mathrm{er}}$ nœud-maturité) sur le rendement du blé et ses principales composantes (cv Top, cases 1982).

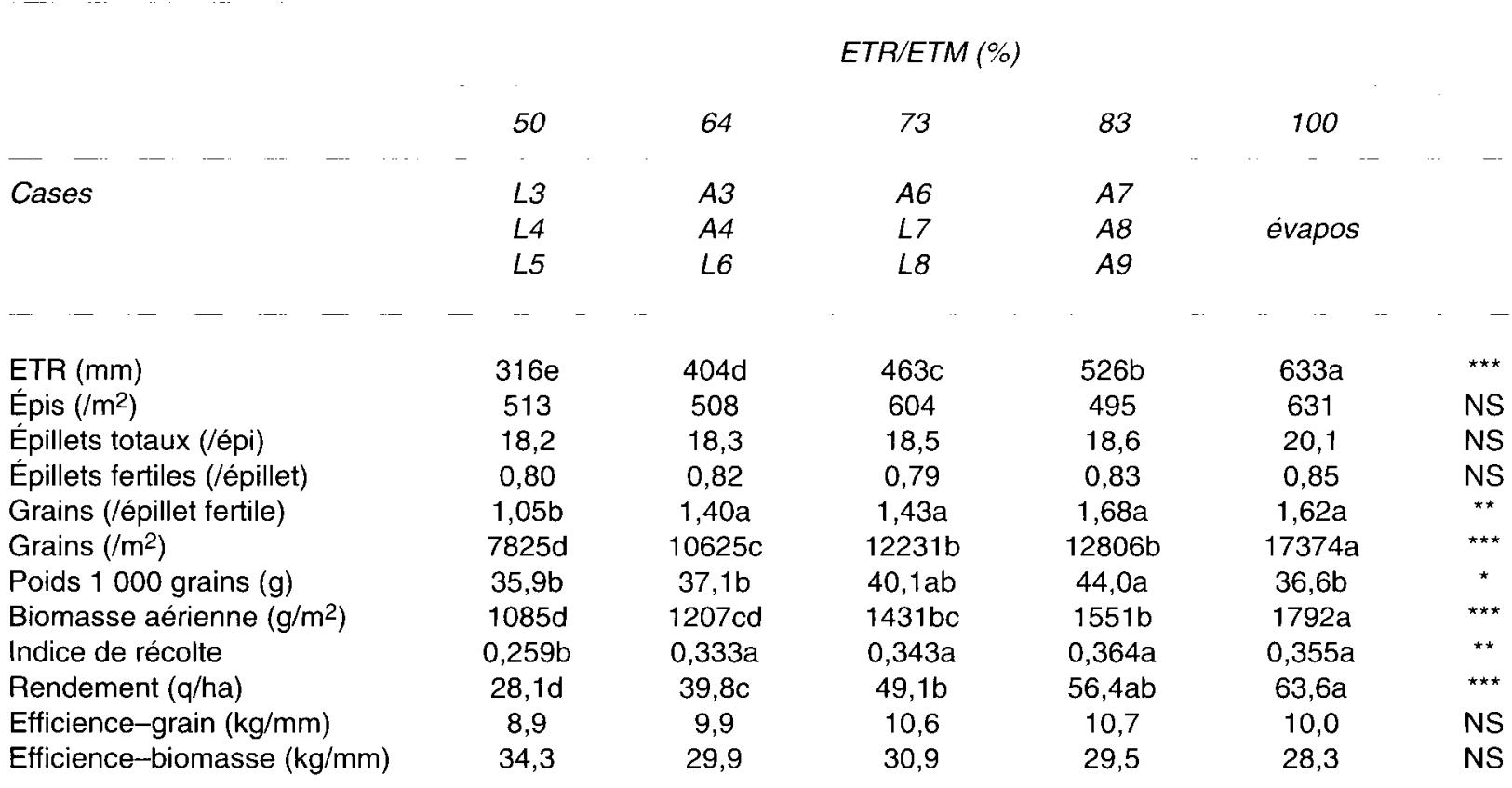

\section{Déficit hydrique dès le début de la montaison (cv Soissons 1994)}

Lorsque la contrainte hydrique est appliquée dès le stade "épi $1 \mathrm{~cm}$ ", l'ensemble des composantes du nombre de grains par $\mathrm{m}^{2}$ est affecté (tableau V). En conditions extrêmes, le nombre d'épillets fertiles par épi et le nombre de grains par épillet fertile sont réduits respectivement de 20 et $35 \%$ par rapport aux valeurs ETM. Le nombre d'épis par $\mathrm{m}^{2}$ n'est affecté significativement qu'en case argileuse peu profonde, où le déficit d'alimentation en eau se manifeste plus rapidement (plus faible RFU). Le nombre d'épillets par épi, déterminé avant l'apparition de la compétition pour l'eau, reste relativement stable, alors que la fertilité des épillets diminue avec la précocité d'apparition du déficit hydrique. En case peu profonde, la réduction de nombre de grains par $\mathrm{m}^{2}$ est de $37 \%$ (limon) et $62 \%$

Tableau V. Effets d'un dessèchement du sol à partir du stade «épi $1 \mathrm{~cm}$ sur le rendement et les principales composantes du blé (cv Soissons, cases 1994) en fonction de la texture et de la profondeur de sol.

\begin{tabular}{|c|c|c|c|c|c|c|c|c|c|c|c|}
\hline & \multicolumn{4}{|c|}{ Argile } & \multicolumn{4}{|c|}{ Limon } & \multirow[t]{3}{*}{ Text } & \multirow[t]{3}{*}{ Prof } & \multirow{3}{*}{$\begin{array}{c}\text { Text } \\
x \\
\text { Prof }\end{array}$} \\
\hline & & -- & - & & & & & & & & \\
\hline & 0,95 & 1,75 & 2,25 & $E T M$ & 0,95 & 1,75 & 2,25 & $E T M$ & & & \\
\hline- & - & & & & & & & & & & \\
\hline ETR/ETM (phase) & 0,54 & 0,63 & 0,65 & 1,00 & 0,51 & 0,70 & 0,72 & 1,00 & - & - & - \\
\hline Épis $\left(/ \mathrm{m}^{2}\right)$ & $476 \mathrm{c}$ & $922 a$ & $929 a$ & $722 b$ & $801 a b$ & $855 a$ & $888 a$ & $709 b$ & * & $* * *$ & *** \\
\hline Épillets totaux (/épi) & $19,2 a$ & $18,4 a b c$ & $18,4 a b c$ & $18,7 a b$ & $17,8 \mathrm{bc}$ & $17,4 \mathrm{c}$ & $17,7 \mathrm{bc}$ & $18,8 \mathrm{a}$ & $\star \star \star *$ & ** & ^ \\
\hline Épillets fertiles (/épillet) & $0,66 \mathrm{~d}$ & $0,70 \mathrm{~cd}$ & $0,72 b c$ & $0,83 a$ & $0,68 \mathrm{~cd}$ & $0,69 \mathrm{~cd}$ & $0,72 b c$ & $0,75 b$ & $\star \star$ & 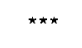 & 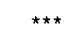 \\
\hline Grains (/épillet fertile) & $1,47 e$ & $1,83 d$ & $1,94 \mathrm{C}$ & $2,13 b$ & $1,44 \mathrm{e}$ & $1,90 \mathrm{c}$ & $2,14 b$ & $2,22 a$ & $\star \star$ & $\star \star \star$ & NS \\
\hline Grains $\left(/ \mathrm{m}^{2}\right)$ & $8958 d$ & $21818 a b$ & $23892 a$ & $23824 a$ & $14054 \mathrm{C}$ & $19314 b$ & $24396 a$ & $22165 a b$ & NS & $\star \star \star \star ~$ & * \\
\hline Poids 1000 grains (g) & $30,7 \mathrm{~b}$ & $26,2 \mathrm{~b}$ & $27,7 \mathrm{~b}$ & $34,0 \mathrm{a}$ & $18,7 \mathrm{c}$ & $25,4 b$ & $28,8 \mathrm{~b}$ & $36,8 \mathrm{a}$ & NS & $* * *$ & *** \\
\hline Biomasse aérienne $\left(\mathrm{g} / \mathrm{m}^{2}\right)$ & $1132 e$ & $1739 \mathrm{bc}$ & 1854ab & $1966 a$ & $1362 d$ & $1605 c$ & $1958 a$ & $1990 a$ & * & $\star \star \star$ & $\star \star$ \\
\hline Indice de récolte & $0,243 e$ & $0,329 \mathrm{c}$ & $0,357 b$ & $0,412 a$ & $0,193 f$ & $0,306 d$ & $0,359 b$ & $0,410 \mathrm{a}$ & $\star \star$ & $\star \star \star ~$ & 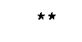 \\
\hline Rendement ( $q /$ ha) & $27,5 \mathrm{e}$ & $57,2 c$ & $66,2 b$ & $81,0 a$ & $26,3 e$ & $49,1 d$ & $70,3 b$ & $81,6 a$ & NS & $\star \star \star$ & * \\
\hline Efficience-grain $(\mathrm{kg} / \mathrm{mm})$ & 10,2 & 17,9 & 20,2 & 16,1 & 10,2 & 13,8 & 19,3 & 16,1 & - & - & - \\
\hline Efficience-biomasse $(\mathrm{kg} / \mathrm{mm})$ & 41,9 & 54,3 & 56,7 & 39,0 & 53,0 & 45,0 & 53,6 & 39,2 & - & - & - \\
\hline
\end{tabular}


(argile) par rapport à l'ETM. Comme le déficit hydrique n'apparaît qu'après floraison en case 2,25 $\mathrm{m}$, on n'observe pas d'effet sur le nombre de grains ; en revanche, en case limoneuse moins profonde $(1,75 \mathrm{~m})$, l'épuisement plus précoce de la réserve se traduit par une baisse de cette composante.

La poursuite du dessèchement en phase de remplissage conduit à la réduction du poids d'un grain avec le rapport ETR/ETM. Le sol argileux $(0,95 \mathrm{~m})$ maintient le poids d'un grain alors que le sol limoneux $(0,95 \mathrm{~m})$ présente un échaudage très marqué. La plus forte limitation du nombre de grains par $\mathrm{m}^{2}$ sur la case argileuse $(-36 \%$ par rapport à $L$ ) a été strictement compensée par le poids d'un grain $(+39 \%)$. Cependant, à rendement égal, le parcours de la case argileuse paraît plus favorable pour la qualité du grain car il aboutit à un poids d'un grain supérieur.

Alors que la biomasse aérienne diffère peu entre cases ETM et 2,25 m, l'indice de récolte est en revanche nettement réduit en situation non irriguée par suite d'une sénescence du couvert plus rapide (épuisement de la ressource après floraison en cases profondes).

En sol argileux, le rendement est moins affecté par la sécheresse qu'en sol limoneux $(0,95$ et $1,75)$; en revanche, lorsque la contrainte hydrique est moins prononcée, le rendement devient supérieur en sol limoneux, en raison d'une moindre résistance au transfert d'eau. Les différences de cessibilité de l'eau conduisent à des parcours d'élaboration du rendement différents selon le type de sol, illustrés par les interactions texture $x$ profondeur souvent très significatives, alors que l'effet de la texture ne l'est pas au seuil $(p<0,05)$. L'efficience de l'eau est très élevée pour les situations de déficit modéré, notamment en sol argileux.

\section{Effets d'une contrainte hydrique marquée selon les phases du cycle (protocole Phases - cv Vizir 1991)}

\section{Déficit durant la phase tallage-début montai- son}

Un défaut d'alimentation hydrique marqué (ETR/ETM voisin de 0,3 en TR et SC) provoque un arrêt précoce de l'émission de talles, notamment en sol argileux. C'est la troisième talle qui est spécialement affectée (tableau VI). Par ailleurs, le nombre d'épillets totaux par épi est réduit de $18 \%$ par rapport aux cases ETM. Le nombre d'épillets fertiles par épi reste inférieur à I'ETM en case argileuse (-14\%) malgré la levée de la contrainte hydrique au stade B2 de l'apex. L'augmentation du nombre de grains par épillet fertile permet cependant de compenser en partie le défaut de nombre de grains.

La reprise des irrigations permet la montée de talles tardives (d'ordre 3), qui représentent $20 \%$ (argile) et $34 \%$ (limon) du potentiel-épis au stade gonflement (résultats non présentés). La récupération du nombre d'épis n'est complète qu'en sol limoneux où le déficit a été plus faible et la levée de la contrainte plus rapide. Le nombre de grains par $\mathrm{m}^{2}$ est ainsi réduit de $17 \%$ (case L) et $20 \%$ (case A) (tableau VII).

La structure plus homogène du peuplementépis et la limitation modérée du nombre de puits (pour un poids de tige peu modifié) assurent un indice de récolte supérieur ou égal à celui de I'ETM pour des conditions hydriques favorables en phase de remplissage. Le plus faible indice foliaire à la floraison (case A) pourrait avoir limité la production d'assimilats après floraison.

\section{Déficit au cours de la phase début montai- son-gonflement}

Des taux de satisfaction du besoin en eau de $60 \%$ (argile) et $76 \%$ (limon) provoquent une réduction de l'indice foliaire (trois dernières feuilles) et du nombre d'épis par $\mathrm{m}^{2}$ (tableau VI). Les résultats sont plus marqués en sol argileux en raison de l'intensité du déficit. Le nombre d'épillets totaux par épi est peu affecté par rapport à l'ETM $(-8 \%)$; en revanche, le nombre d'épillets fertiles par épillet est davantage réduit $(-14 \%)$, notamment à la base de l'épi. Le nombre de grains par épillet fertile est réduit par rapport à l'ETM (-9\%) malgré la levée de la contrainte hydrique au gonflement. En conséquence, et malgré la réduction de nombre d'épis, le nombre de grains par épi est réduit de $19 \%$ par rapport à I'ETM. Par ailleurs, la régression des talles végétatives est de $28 \%$ en case argileuse ; celle-ci est en partie compensée par une montée tardive de talles après épiaison, soit $42 \%$ de la population d'épis totaux en case $A$ et $14 \%$ en case $\mathrm{L}$. Contrairement au traitement TR, l'intérêt de cette montée décalée est marginal pour le rendement, en raison de la faible hauteur des tiges, de la faible fertilité et de la maturation tardive de l'épi. Le plus faible nombre de grains par $\mathrm{m}^{2}(-44 \%$ par rapport à l'ETM en $\mathrm{A})$ permet d'assurer un bon remplissage $(+13 \%$ de $P$ $1000 \mathrm{~g})$ et donc une valeur d'IR proche de I'ETM 
Tableau VI. Effets d'une contrainte hydrique d'intensité variable appliquée à différentes phases du cycle du blé sur l'élaboration du nombre de grains par $\mathrm{m}^{2}$ (cv Vizir, cases 1991).

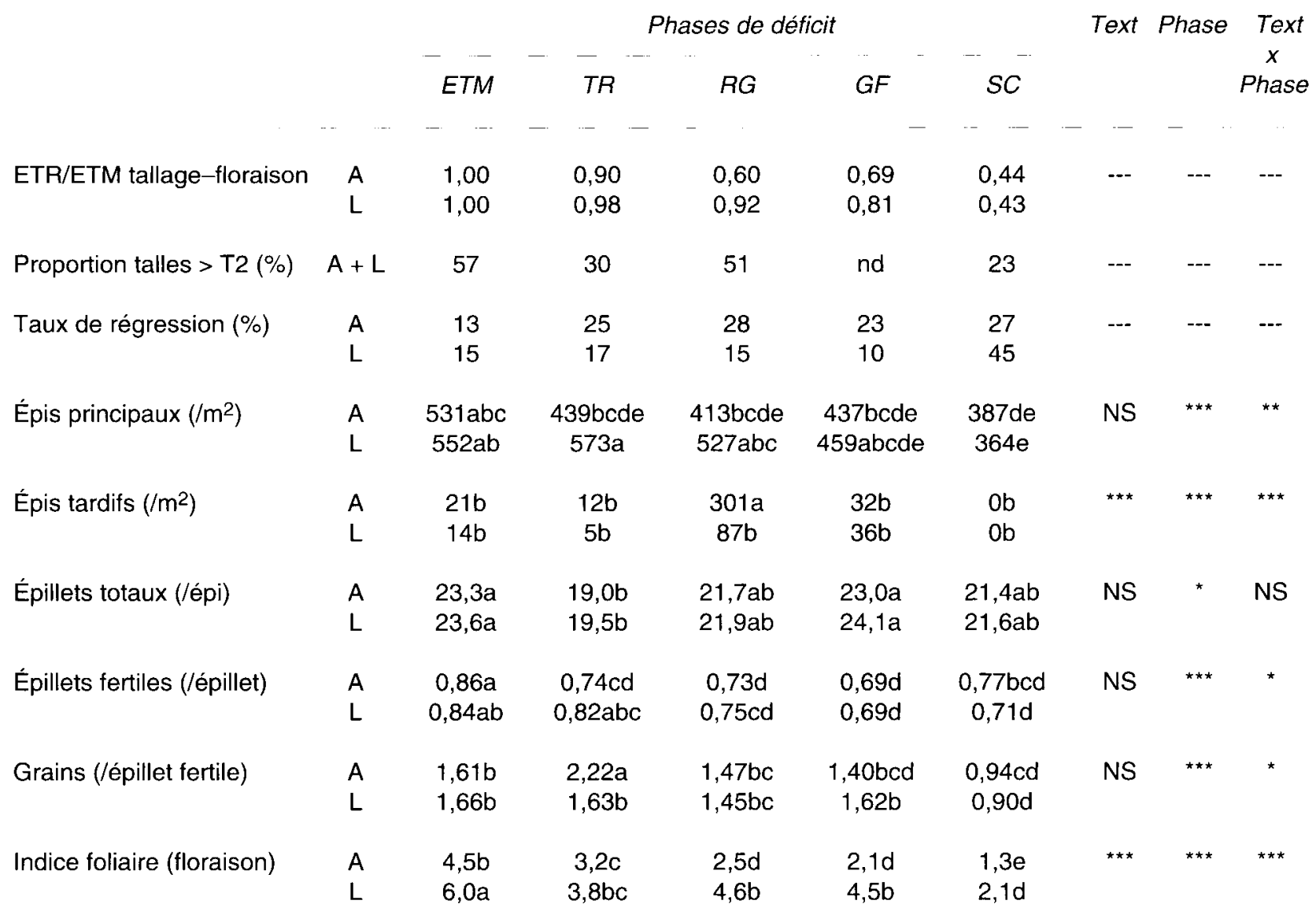

Épis principaux : épis constituant la strate dominante du peuplement. Épis tardifs : épis issus de talles montées après retour des irrigations + épiochons.

Tableau VII. Effets d'une contrainte hydrique d'intensité variable appliquée à différentes phases du cycle du blé sur l'élaboration du rendement (cv Vizir, cases 1991) - épis tardifs non compris.

\begin{tabular}{ccccccccc}
\multicolumn{4}{c}{ Phases de déficit } & & \multicolumn{3}{c}{ Text Phase Text } \\
- & & - & & & & & & $x$ \\
ETM & TR & RG & GF & FSL & SLM & SC & Phase
\end{tabular}

\begin{tabular}{|c|c|c|c|c|c|c|c|c|c|c|}
\hline Grains $\left(/ \mathrm{m}^{2}\right)$ & $\begin{array}{l}A \\
L\end{array}$ & $\begin{array}{c}17131 \mathrm{a} \\
18115 \mathrm{a}\end{array}$ & $\begin{array}{c}13737 b c \\
14971 b\end{array}$ & $\begin{array}{c}9641 d \\
12775 c\end{array}$ & $\begin{array}{c}9720 d \\
12222 c\end{array}$ & $\begin{array}{c}12176 c \\
13242 b c\end{array}$ & $\begin{array}{c}14016 b c \\
14517 b\end{array}$ & $\begin{array}{l}6238 e \\
5046 e\end{array}$ & $\star \star \star$ & $\star \star \star$ \\
\hline Poids 1000 grains $(\mathrm{g})$ & $\begin{array}{l}A \\
L\end{array}$ & $\begin{array}{c}37,0 \mathrm{~cd} \\
35,7 \mathrm{~d}\end{array}$ & $\begin{array}{l}38,0 \mathrm{~cd} \\
36,9 \mathrm{~cd}\end{array}$ & $\begin{array}{c}41,8 b c \\
39,2 c\end{array}$ & $\begin{array}{l}50,0 a \\
45,7 b\end{array}$ & $\begin{array}{l}38,6 \mathrm{~cd} \\
37,6 \mathrm{~cd}\end{array}$ & $\begin{array}{l}37,7 \mathrm{~cd} \\
38,1 \mathrm{~cd}\end{array}$ & $\begin{array}{l}42,8 b \\
43,6 b\end{array}$ & $\star \star \star \star ~$ & $\star \star \star \star$ \\
\hline Biomasse aérienne $\left(\mathrm{g} / \mathrm{m}^{2}\right)$ & $\begin{array}{l}A \\
L\end{array}$ & $\begin{array}{l}1528 a \\
1530 a\end{array}$ & $\begin{array}{l}1276 c \\
1269 c\end{array}$ & $\begin{array}{l}1010 d \\
1556 a\end{array}$ & $\begin{array}{l}1237 \mathrm{c} \\
1540 \mathrm{a}\end{array}$ & $\begin{array}{l}1391 b \\
1435 b\end{array}$ & $\begin{array}{c}1404 b \\
1483 a b\end{array}$ & $\begin{array}{l}849 e \\
768 e\end{array}$ & 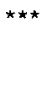 & $* \star *$ \\
\hline Rendement ( $q / h a)$ & $\begin{array}{l}A \\
L\end{array}$ & $\begin{array}{l}63,4 a \\
64,7 a\end{array}$ & $\begin{array}{c}52,2 \mathrm{bc} \\
55,2 \mathrm{~b}\end{array}$ & $\begin{array}{l}40,3 d \\
50,1 c\end{array}$ & $\begin{array}{c}48,6 \mathrm{~cd} \\
55,9 \mathrm{~b}\end{array}$ & $\begin{array}{c}47,0 \mathrm{~cd} \\
49,8 \mathrm{c}\end{array}$ & $\begin{array}{c}52,8 b c \\
55,3 b\end{array}$ & $\begin{array}{l}26,7 e \\
22,0 e\end{array}$ & $\star \star \star$ & $\star \star \star$ \\
\hline Indice de récolte & $\begin{array}{l}A \\
L\end{array}$ & $\begin{array}{c}0,415 b c \\
0,423 b\end{array}$ & $\begin{array}{c}0,409 b c \\
0,435 a\end{array}$ & $\begin{array}{c}0,399 \mathrm{bcd} \\
0,322 \mathrm{f}\end{array}$ & $\begin{array}{c}0,393 \mathrm{bcd} \\
0,363 \mathrm{de}\end{array}$ & $\begin{array}{l}0,338 \mathrm{e} \\
0,347 \mathrm{e}\end{array}$ & $\begin{array}{c}0,376 \mathrm{bcd} \\
0,373 \mathrm{de}\end{array}$ & $\begin{array}{l}0,315 f \\
0,287 f\end{array}$ & $\star \star \star \star ~$ & $\star \star \star$ \\
\hline
\end{tabular}


en cases argileuses (tableau VII). Au cours de cette phase, où l'organogenèse est très dépendante de la croissance végétative, il est logique que la contrainte hydrique affecte peu l'indice de récolte. Si l'on tient compte des talles tardives dans le poids de paille, IR chute très nettement $(0,35$ en case $A)$.

En limon, les valeurs d'IR sont généralement plus faibles en phase végétative en raison d'une verse à la floraison (reprise de l'irrigation intense) qui a affecté les traitements $R G$ et GF.

\section{Déficit au cours de la phase gonflement- floraison}

Le rapport ETR/ETM n'a pû être abaissé sévèrement pour les traitements GF $(0,78$ en limon, 0,89 en argile) (tableau III). Les indices foliaires actifs à la floraison sont pourtant notablement réduits par rapport à l'ETM par suite d'une sénescence précoce des dernières feuilles $(-25 \%$ en limon, $-53 \%$ en argile) (tableau VI). Le nombre d'épillets totaux par épi est logiquement voisin de I'ETM alors que le nombre d'épillets fertiles par épillet est sévèrement réduit $(-19 \%)$. En limon, le nombre de grains par épillet fertile n'est pas affecté ; en argile, la réduction est de $13 \%$. La régression des talles est moins prononcée qu'en TR et RG mais la compensation par des montées tardives de talles est moins efficace que précédemment. Le nombre de grains par $\mathrm{m}^{2}$ est réduit de $33 \%$ (case L) et $43 \%$ (case A) par rapport à I'ETM, ce qui se traduit par un poids d'un grain particulièrement élevé $(+28 \%$ en $L$ et $+35 \%$ en A par rapport à l'ETM) (tableau VII). La concurrence avec les talles tardives lors du remplissage est probablement plus faible qu'en RG. La réduction du nombre de puits et le faible indice foliaire à la floraison (sénescence précoce), alors que la biomasse végétative est peu affectée, conduisent à un indice de récolte plus faible, malgré de bonnes conditions de remplissage.

\section{Déficit hydrique en post-floraison}

\section{Phase floraison-stade laiteux}

Le déficit créé sur ces cases peu profondes est très intense en argile $(0,28)$, plus modéré en limon $(0,69)$ (tableau III) ; il se traduit par un avortement de grains important $(-28 \%)$. L'examen du poids spécifique du grain montre peu de différences entre traitements et aucun signe d'échaudage (PS $>80 \mathrm{~kg} / \mathrm{hl}$ ), les températures ayant rarement dépassé $28^{\circ} \mathrm{C}$ lors du remplissage du grain. Le faible poids de 1000 grains traduirait plutôt un défaut d'alimentation carbonée lors de la formation des enveloppes du grain. La sénescence précoce a limité l'activité photosynthétique du couvert malgré la reprise des irrigations au stade laiteux. C'est pourquoi on observe une chute prononcée de l'indice de récolte $(0,34$ pour $F S L$ contre 0,42 pour ETM).

\section{Phase stade laiteux-maturité}

Des valeurs d'ETR/ETM de 0,51 en argile et de 0,60 en limon après le stade laiteux provoquent également une baisse du poids d'un grain et de l'indice de récolte, certes plus modérée qu'en FSL, la taille du grain n'étant pas affectée. Le plus faible nombre de grains par rapport à l'ETM ne peut s'expliquer que par un arrêt trop précoce des irrigations (dès la floraison) et un avortement de grains.

Les bilans de biomasse, effectués entre floraison et maturité, montrent que le taux de remobilisation des réserves de la tige est plus important en condition de déficit hydrique post-floraison (21\% en SLM et $29 \%$ en SC contre $11 \%$ à I'ETM). Comme l'assimilation nette est faible au cours du remplissage, la contribution des réserves au poids du grain est plus forte $(52 \%$ en SLM et $96 \%$ en SC contre $20 \%$ à l'ETM). Ce processus contribue au maintien d'un indice de récolte minimum $(0,30)$ et d'un poids de 1000 grains proche du potentiel $(43 \mathrm{~g})$ pour les conditions de très faible assimilation en post-floraison (cases SC).

\section{Récupération d'une contrainte hydrique de durée variable avant floraison} (protocole Récupération - cv Soissons 1994)

La reprise de l'irrigation après une période de déficit hydrique survenant avant floraison permet une compensation du nombre d'épis par la montée de talles tardives (tableau VIII). En 1991, la contribution de ces talles au rendement n'avait pas été évaluée. En 1994, dans le cas d'une sécheresse semis-floraison (SF), la part des épis tardifs représente $38 \%$ du peuplement-épis (tableau VIII) ; ce phénomène est moins prononcé pour le traitement $S G$ pour lequel il n'a pas été possible de distinguer les deux populations de talles à maturité. Cette montée tardive permet d'obtenir en SF un peuplement-épis double de celui du témoin ETM. Le gain de peuplementépis augmente avec la durée du déficit en eau. En dépit d'un certain rattrapage du nombre de grains par $\mathrm{m}^{2}$, le rendement $n^{\prime} e s t$ pas compensé 
Tableau VIII. Effets de la durée du déficit hydrique avant floraison sur le rendement du blé et ses principales composantes (cv Soissons, cases 1994).

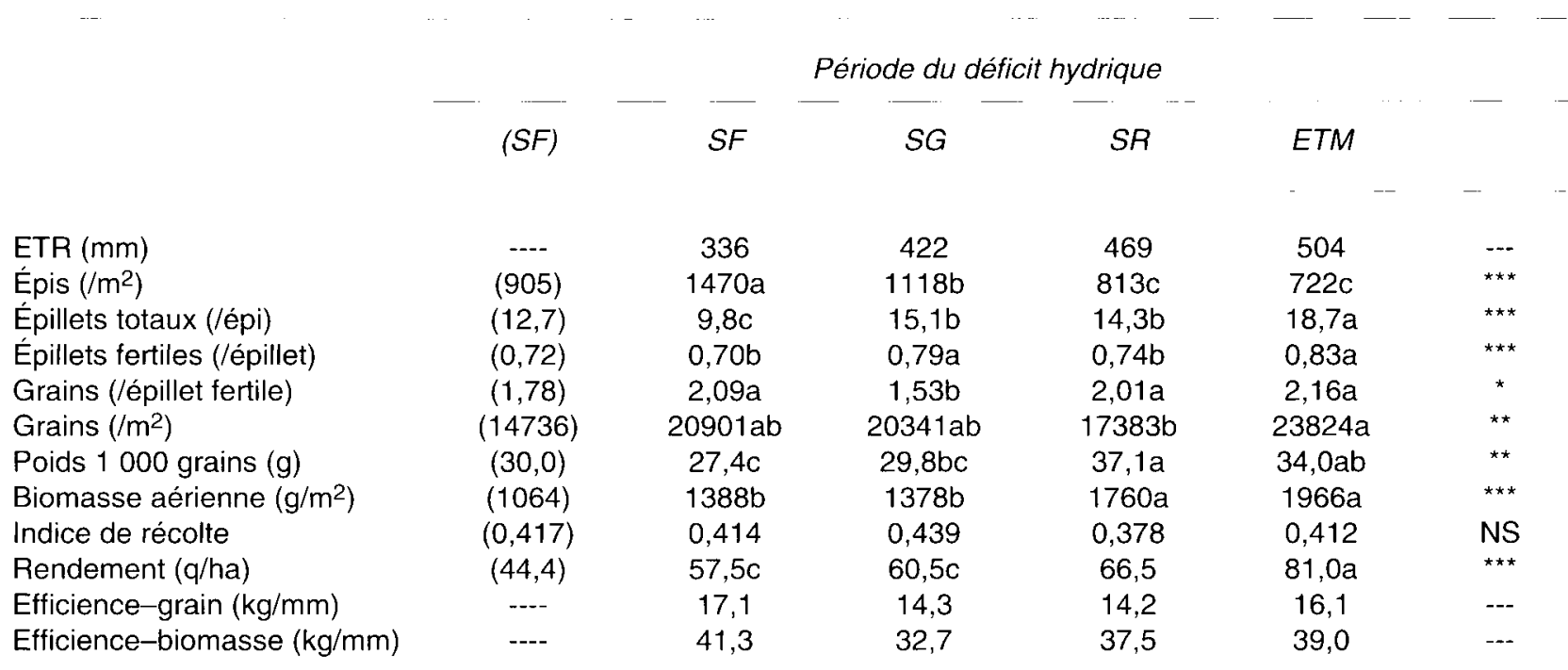

Entre parenthèses, sans prise en compte des talles reproductrices tardives.

totalement en raison d'un poids de 1000 grains inférieur aux conditions de l'ETM : le décalage de la période de remplissage vers des conditions plus échaudantes en est la cause. D'après Masse et al (1989), sans déficit hydrique, le défaut de poids de 1000 grains par rapport au potentiel de la variété augmente de 0,7 g par jour où la température maximale dépasse $25^{\circ} \mathrm{C}$, ce qui est cohérent avec le décalage de maturation vers les périodes de fortes températures observé en SF et SG (+15 jours après ETM). L'indice de récolte, plus faible sur les talles tardives, est maximal en SG (équilibre entre rationnement précoce et bonne alimentation post-floraison). Le rendement diminue avec la durée d'action et l'intensité de la contrainte hydrique. L'efficience de l'eau exprimée pour la production de grain diminue avec la durée du déficit hydrique.

\section{Essai de synthèse : réponse du blé à la contrainte hydrique}

Nous proposons une approche synthétique de la réponse du blé à la contrainte hydrique intégrant l'ensemble des situations analysées plus haut.

\section{Réponse du rendement}

Tous génotypes et traitements confondus (fig 2), le rapport des rendements ( $\left.\mathrm{Rdt}_{\mathrm{ETR}} / \mathrm{Rdt}_{\mathrm{ETM}}\right)$ augmente de manière quasi proportionnelle avec le taux de satisfaction des besoins en eau (ETR/ETM) sur l'ensemble du cycle du blé ( $r=$ $0,836)$. La valeur d'évaporation du sol déduite pour un rapport $\mathrm{Rdt}_{E T R} / \mathrm{Rdt}_{\mathrm{ETM}}$ nul et une ETM de $570 \mathrm{~mm}$ en moyenne est de $49 \mathrm{~mm}$.

En deçà d'un rapport ETR/ETM de 0,65, la chute de rendement s'accentue, notamment en petits fûts (1982) et en cases peu profondes (A95 et L95 - 1994) où le dessèchement du sol est brutal. En revanche, une sécheresse continue et progressive (SC - 1991) n'affecte pas le rendement de manière aussi drastique que la relation linéaire pouvait le suggérer. On remarque également qu'un dessèchement progressif en sol profond (A225 et L225 - 1994), qui affecte le blé plus tardivement, diminue moins le rendement que des stress en phase de montaison (SF et SG - 1994). Enfin, ce sont les stress hydriques autour de la floraison (GF et FSL - 1991) qui affectent le rendement de manière très prononcée. II n'apparaît pas d'effet variétal évident.

Ces écarts à la droite illustrent les différences de sensibilité des différentes phases au déficit hydrique, que l'on peut mieux classer en utilisant le rapport ETR/ETM par phase (fig 3). Ainsi, une valeur d'ETR/ETM de 0,5 maintenue au cours du tallage (TR), de la montaison (RG, GF) ou du remplissage (SLM, FM) diminue le rendement de 4,48 et $22 \%$ respectivement. Les situations de stress continu (SC 1991, Dessèchement 1982 et 1994), affectées dès la montaison, s'alignent sur les stress pré-floraison. Si l'on ne tient pas comp- 


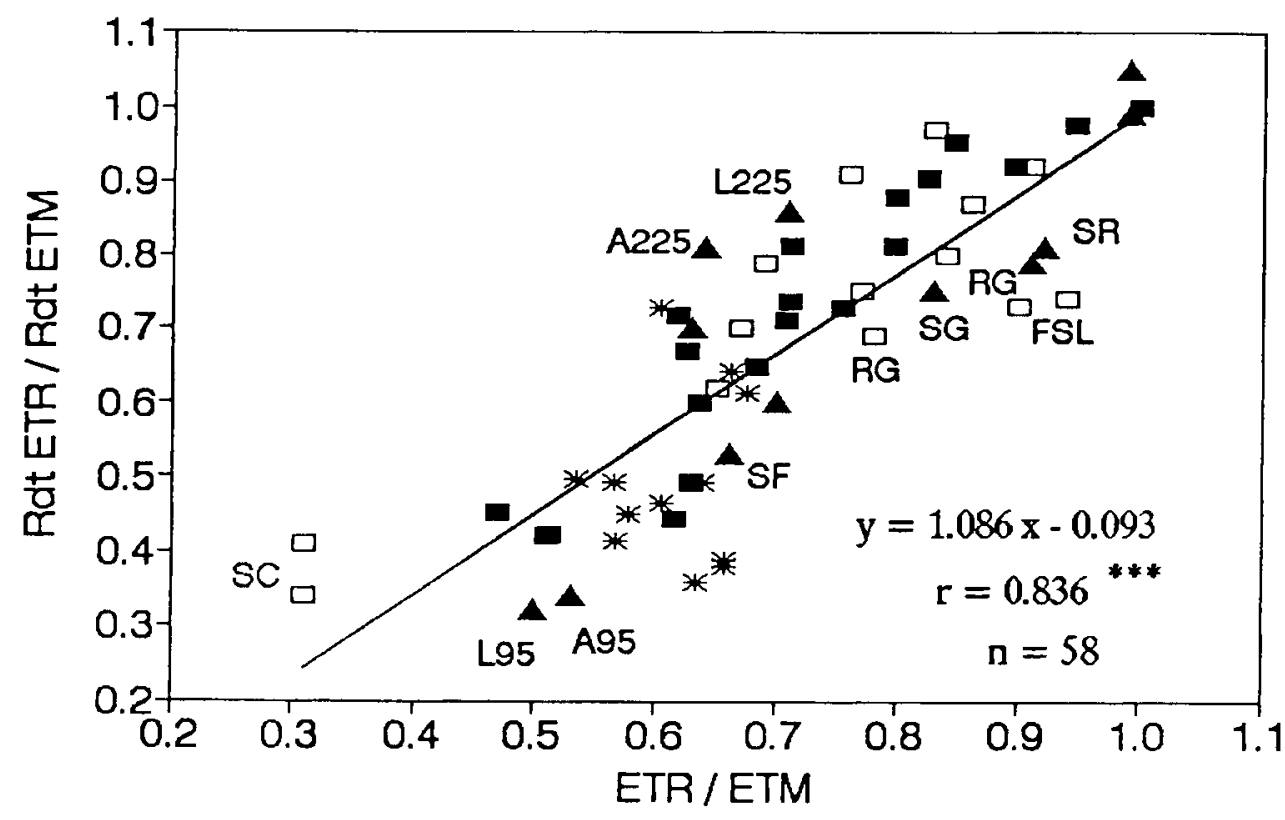

$\begin{array}{ccccc}\text { Top (cases) } & * & \text { Top (fûts) } & \square & \text { Vizir } \\ 1982 & & 1982 & & 1991\end{array}$

te de la production des talles tardives, les phases RG, FSL et surtout GF apparaissent comme les plus sensibles au défaut d'alimentation hydrique.

Les variations d'efficience de l'eau exprimée pour la production de grain traduisent également des déséquilibres dans l'utilisation de la ressour-

\section{- Soissons 1994}

Fig 2. Relation entre le rapport des rendements (Rdt ETR $_{\text {Th }} /$ Rdt $_{\text {ETM }}$ ) et le degré de satisfaction des besoins en eau de la levée à la maturité du blé (ETR/ETM). ce au cours du cycle. Alors que l'efficience de l'eau varie peu en cases profondes pour la variété Top (tableau IV), des différences marquées apparaissent en petits fûts $(5,7$ à $12,1 \mathrm{~kg} / \mathrm{mm})$ pour des valeurs d'ETR voisines $(380-430 \mathrm{~mm})$. Étant donné les différences de cessibilité de l'eau

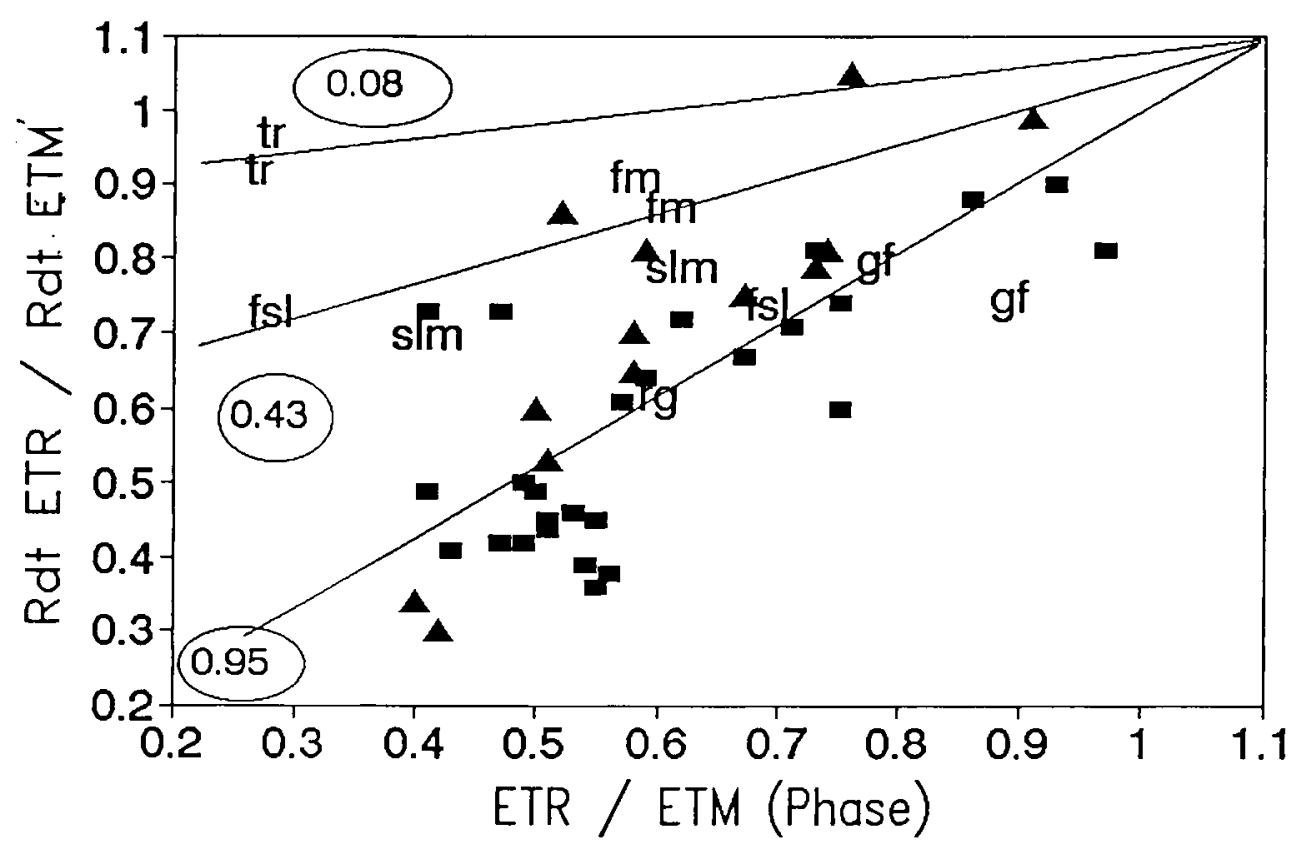

(xy) Vizir 1991 - Top 1982 - Soissons 1994
Fig 3. Relation entre le rapport des rendements $\left(\mathrm{Rdt}_{\mathrm{ETR}} / \mathrm{Rdt}_{\mathrm{ETM}}\right)$ et le degré de satisfaction des besoins en eau par phase (ETR/ETM). 
des sols considérés (Puech et al, 1977), il est logique de s'attendre à des variations de la vitesse de consommation du stock initial par le blé. Les variations d'Eff-Gr dépendent en effet de la part de l'ETR consommée avant épiaison (fig 4) : les sols légers (SL et SLA), qui présentent une forte cessibilité de l'eau, entraînent un épuisement prématuré des réserves, préjudiciable à EffGr. À l'inverse, le sol argileux (ASL), qui contraint le blé au cours de la montaison par une plus forte rétention d'eau, assure une alimentation profonde suffisante après l'épiaison, ce qui se traduit par une meilleure efficience de l'eau. Cette conclusion s'applique également aux cases 1994 (protocole Dessèchement). Par ailleurs, l'efficience de l'eau est maximale pour des déficits hydriques continus mais modérés (ETR/ETM de 0,65 à 0,75 ), alors que les valeurs d'Eff-Gr sont plus faibles à l'ETM et pour des contraintes hydriques marquées et brutales.

\section{Réponse du nombre de grains par $\mathrm{m}^{2}$}

De la même manière, on peut rendre compte de l'effet de la contrainte hydrique sur le nombre de grains par $\mathrm{m}^{2}$ en croisant $\mathrm{NG}_{\mathrm{ETR}} / \mathrm{NG}_{\mathrm{ETM}}$ avec ETR/ETM (fig 5). La relation obtenue est très étroite $(r=0,899)$ et de pente plus prononcée que pour le rendement, ce qui illustre la forte sensibilité du nombre de grains au déficit hydrique. Ainsi, une relation unique peut être retenue, contrairement au rendement, pour prédire la diminution du nombre de grains par $\mathrm{m}^{2}$, quelle que soit la phase affectée par le déficit hydrique. L'effet variétal ne joue pas sur cette relation.

\section{Réponse de la biomasse et de l'indice de récolte}

La biomasse aérienne est moins affectée que le rendement par le déficit hydrique (fig 6) ; si la relation entre $\mathrm{Rdt}_{\text {ETR }} / \mathrm{Rdt}_{\mathrm{ETM}}$ et $M_{\text {ETR }} /$ MST $_{E T M}$, toutes situations confondues, est assez étroite pour des stress modérés, le rapport des rendements chute plus brusquement pour la plupart des points en deçà de 0,75 . Seuls les traitements "récupération" (SF et $S G$ - 1994) et "stress continu» (SC - 1991) ne sont pas autant affectés et suivent la bissectrice. Alors que le rapport des indices de récolte $\left(I R_{E T R} / I R_{E T M}\right)$ est peu affecté par des stress modérés, la chute est brutale pour des valeurs d'ETR/ETM inférieures à 0,75 (fig 7). En revanche, les situations SC conservent un indice de récolte égal à $70 \%$ de la valeur ETM malgré un taux de satisfaction du besoin hydrique voisin de $30 \%$. À l'inverse, IR chute brutalement dans
Fig 4. Relation entre l'efficience de l'eau exprimée pour la production de grain et la part de l'eau consommée avant épiaison du blé (cv Top 1982). S (sable), SL (sable limoneux), LA (limon argileux), Al (argile lourde), ASL (sol Al/sol $\mathrm{SL}$ ), SLA (sol SL/sol Al).

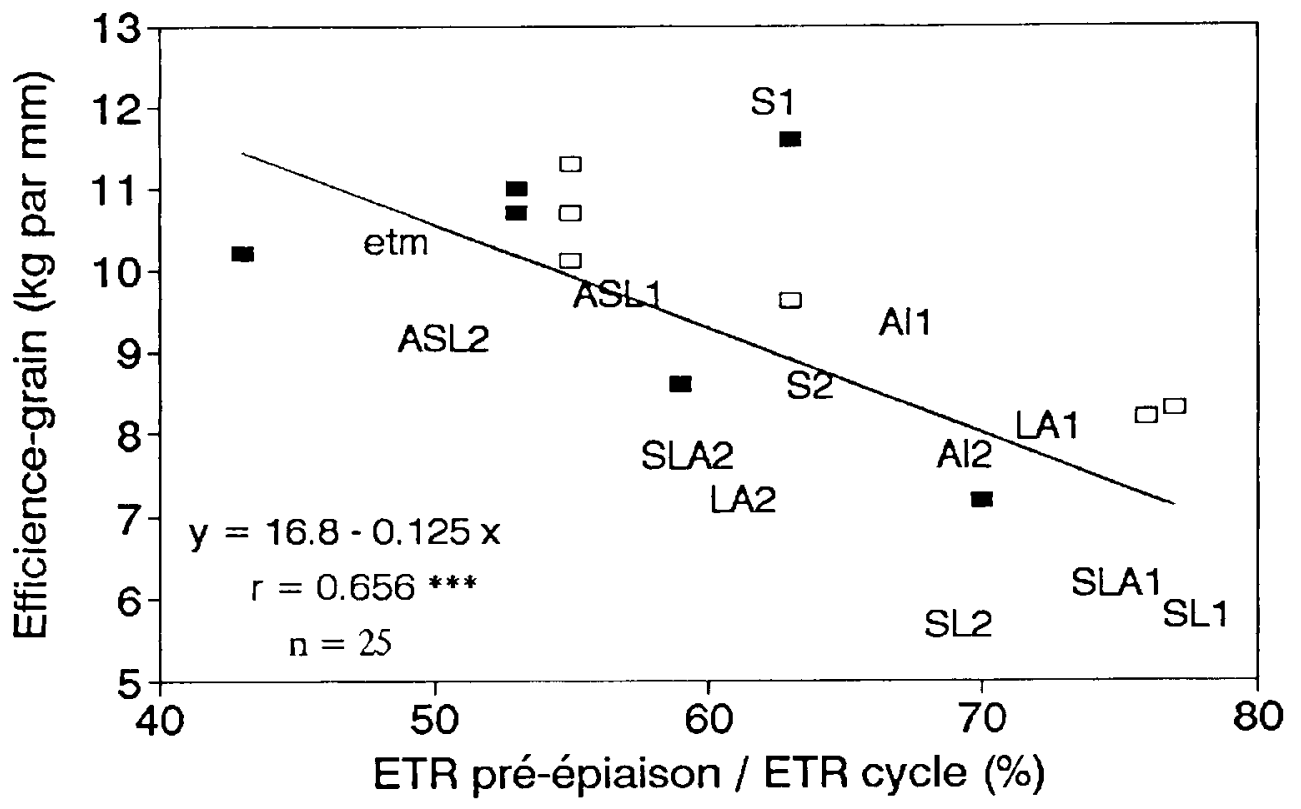

(xi) fûts $\quad$ - cases argile (A) $\square$ cases limon (L)

$\mathrm{x}=$ type de sol etm = évapotranspiromètre

$\mathrm{i}=$ répétition 


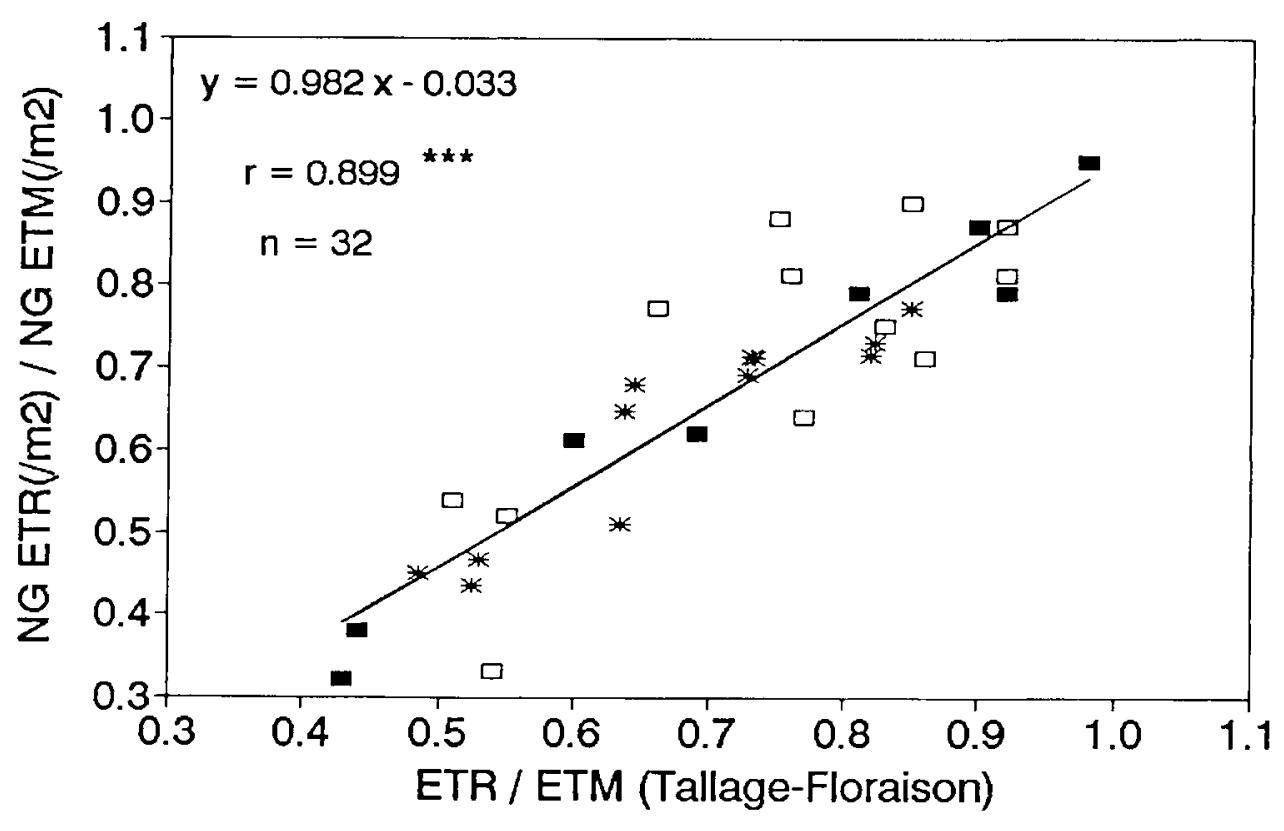

* Top 1982
- Vizir 1991

\section{ㅁ Soissons 1994}

Fig 5. Relation entre le rapport des nombres de grains par $\mathrm{m}^{2}$ $\left(N G_{E T R} / N G_{E T M}\right)$ et le degré de satisfaction des besoins en eau de la levée à la maturité du blé (ETR/ETM). le cas du traitement FSL, ce qui suggère l'existence pour cette variable d'une phase sensible peu après floraison.

La répartition de l'eau consommée (ETR) au cours du cycle conditionne la valeur de l'indice de récolte. La relation est en effet positive entre IR et la part de I'ETR consommée après floraison, avec un plafonnement vers 30-35\% de I'ETR (fig 8). Cette contribution, suffisante après floraison, correspond à des situations irriguées au cours du remplissage, aux cas de rationnement avant floraison ou aux cases profondes non irriguées, qui permettent un report de la consommation hydrique (allongement de la durée de remplissage du grain). Les situations où la croissance végétative n'a pas été rationnée avant floraison (case L95, fûts 1982) se traduisent à l'inverse par un échaudage prononcé, lié à l'excès

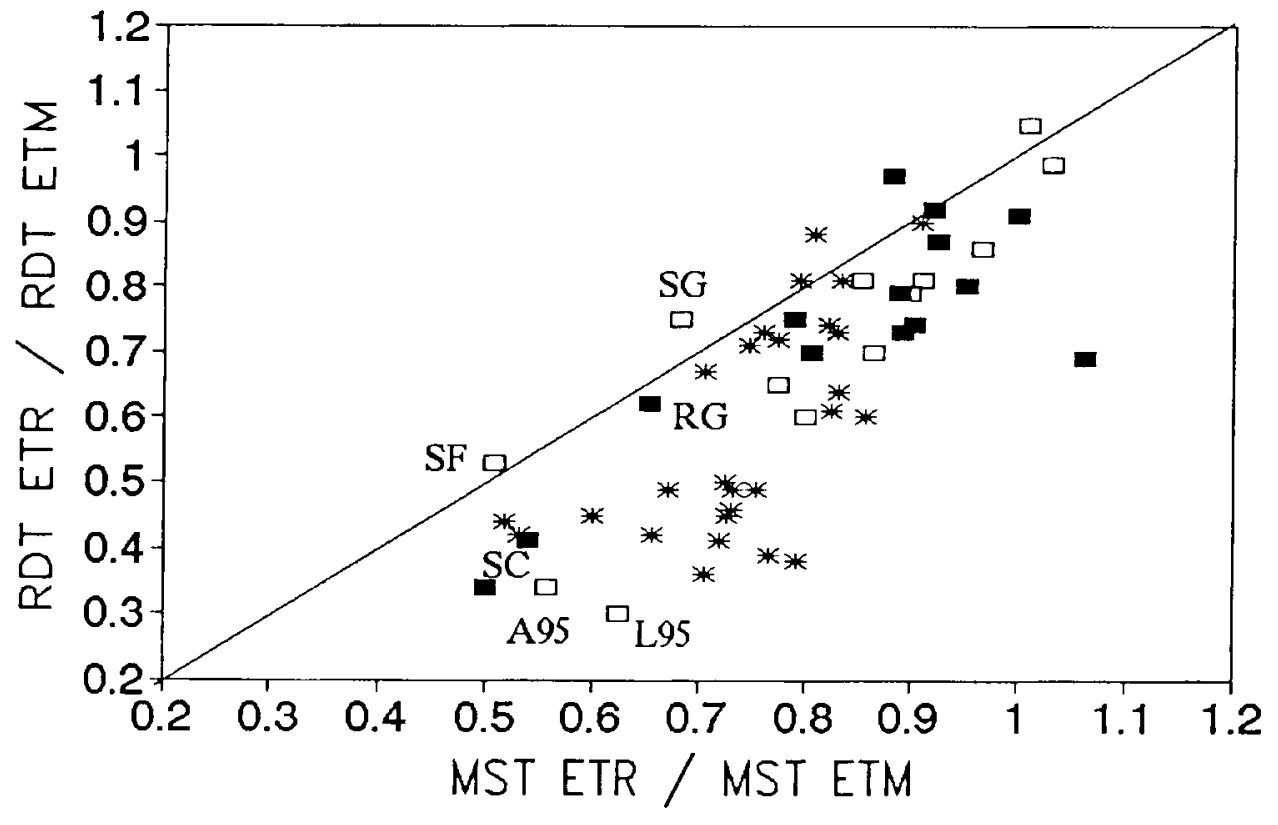

* Top 1982
- Vizir 1991
ㅁ Soissons 1994
Fig 6. Relation entre le rapport des rendements ( $\operatorname{Rdt}_{\mathrm{ETR}} / \mathrm{Rdt}_{\mathrm{ETM}}$ ) et des biomasses aériennes (MST ETR $_{\text {MST }} /$ ETM . 
Fig 7. Relation entre le rapport des indices de récolte $\left(\mathrm{I}_{\mathrm{ETR}} / \mathrm{R}_{\mathrm{ETM}}\right)$ et le degré de satisfaction des besoins en eau de la levée à la maturité du blé (ETR/ETM).

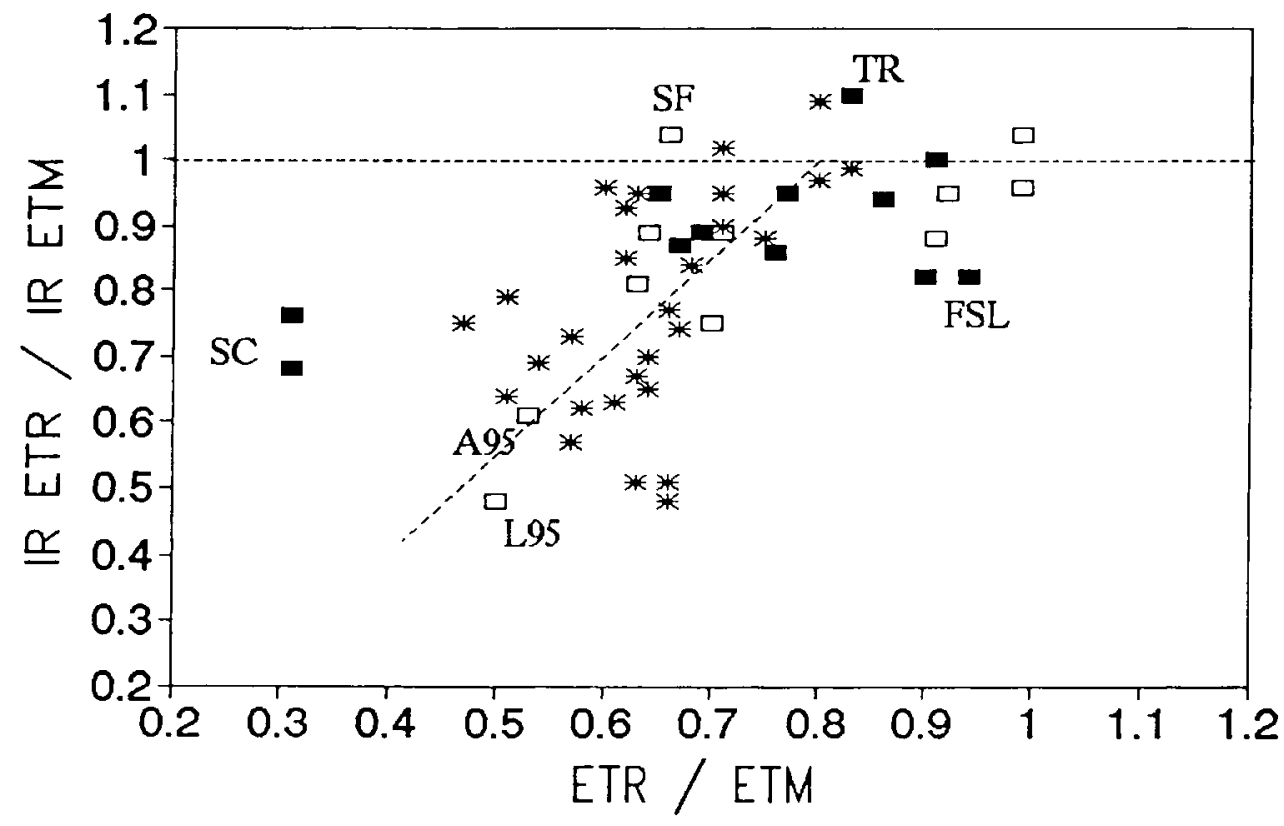

* Top 1982
- Soissons 1994 de végétation et à l'épuisement précoce des réserves, qui diminue IR de manière drastique.

\section{Modèle simple d'effet de la contrainte hydrique sur l'indice de récolte}

L'effet d'une contrainte hydrique variant par son intensité, sa durée et sa période d'action sur l'in- dice de récolte du blé peut être décrit par un modèle simple.

Le nombre de jours de stress hydrique (NJS) est un indicateur de l'intensité et de la durée de la contrainte hydrique (Hiler et Clark, 1971). On peut l'exprimer de la manière suivante, pour une phase $p$, de durée $\mathrm{NJ}$ (en jours) :

$$
\mathrm{NJS}_{p}=N J_{p}\left(1-E T R_{p} / \mathrm{ETM}_{p}\right)
$$

Fig 8. Relation entre l'indice de récolte et l'eau consommée après floraison (rapportée à I'ETR du cycle).

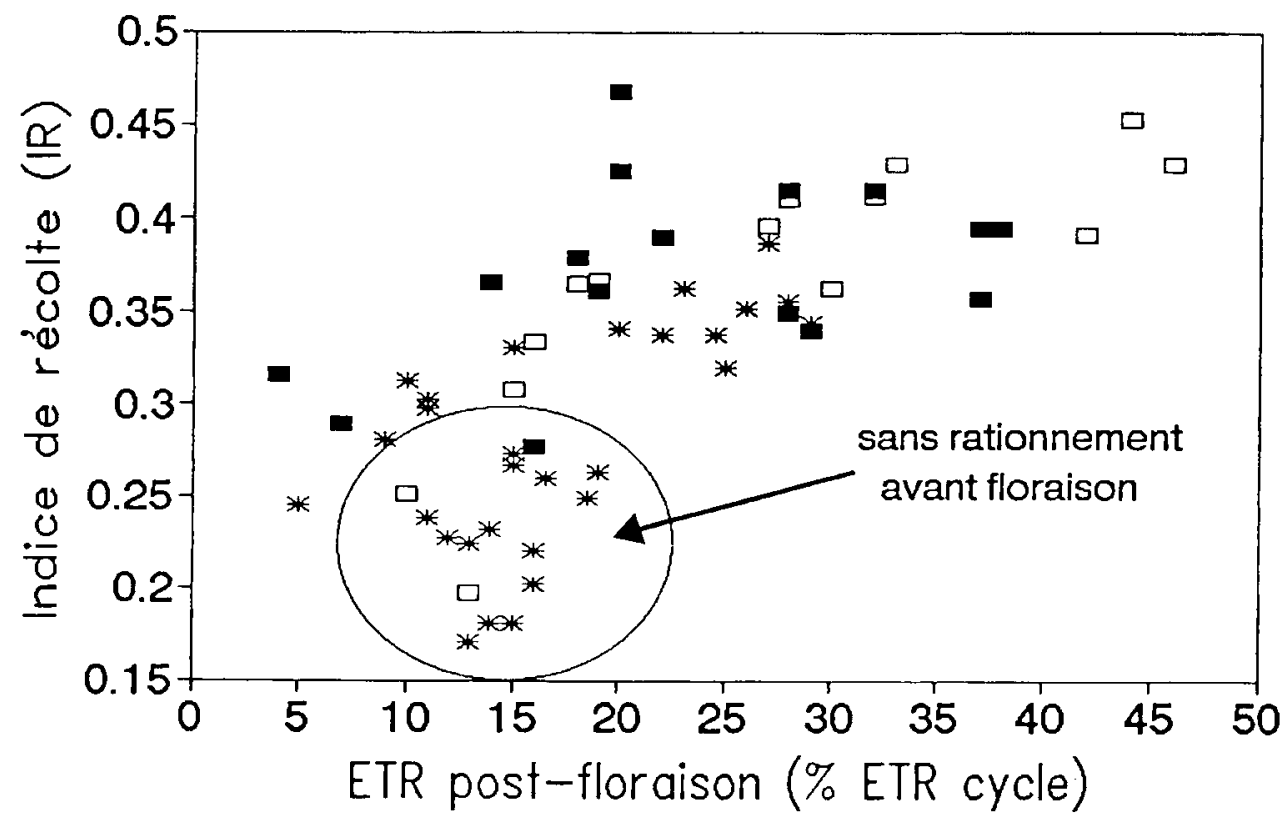

* Top 1982 


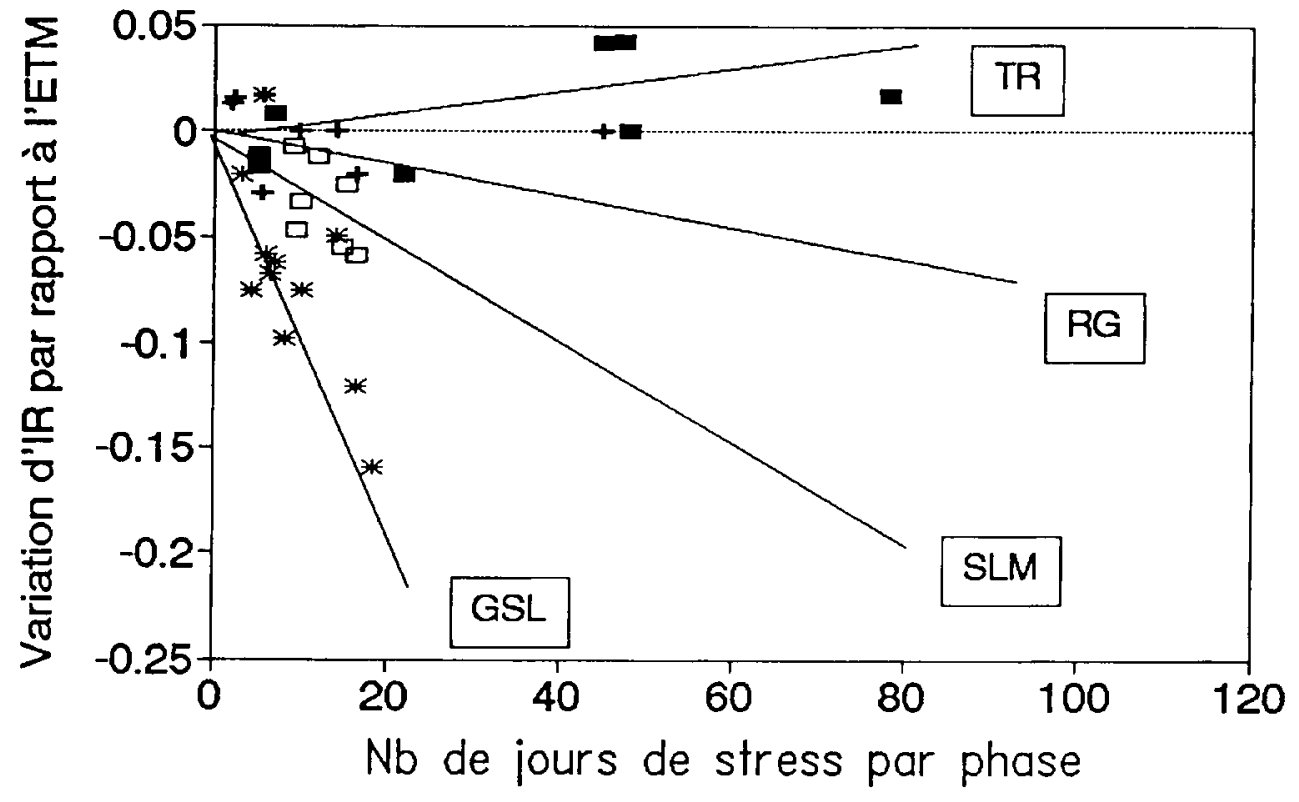

- Tal-Red + Red-Gonf * Gonf-SLait $\square$ Remp
Fig 9. Relation entre la variation d'indice de récolte par rapport à I'ETM et le nombre de jours de stress hydrique par phase. Données issues du protocole Phases (1991 et 1994) et extraites de Blackwell et Innes (1981) et Mongensen et al (1985).
La variation d'indice de récolte par rapport à la valeur observée à l'ETM a été mise en relation avec l'indice NJS $p$ pour les dispositifs "Phases" 1991 et 1994 (fig 9). La pente $p_{n}$ exprime la variation d'indice de récolte par jour de stress. On considère, par exemple, qu'un jour de stress où la croissance est nulle $(E T R / E T M=0)$ équivaut à dix jours où ETR/ETM est de 0,9 . Étant donné le petit nombre de résultats disponibles par phase, les valeurs obtenues par Innes et Blackwell (1981) et Mogensen et al (1985) en lysimètres, sous climat moins contraignant et pour d'autres variétés, ont été utilisées pour le paramétrage. La mise au point de ces relations devrait permettre d'affiner la simulation du rendement du blé par le modèle Epic, comme cela a été proposé pour le maïs (Quinones et Cabelguenne, 1990).

Une contrainte hydrique lors de la phase TR permet d'augmenter légèrement l'indice de récolte par le biais d'un rationnement végétatif (pente $p 1=0,510^{-3}$ ). Pour les autres phases, l'effet est dépressif : IR diminue avec NJSp selon les pentes $p 2=0,810^{-3}(\mathrm{RG}), p 3=-1010^{-3}$ (GF et FSL) et $p 4=-2,510^{-3}$ (SLM). Ces données confirment la sensibilité particulière de la phase gonflement-stade laiteux au défaut d'alimentation hydrique, l'effet positif d'un stress au cours de la phase tallage-début montaison, enfin le comportement intermédiaire des autres phases. La valeur de ces pentes n'est qu'une base de départ pour la simulation. Les pentes $p 1$ et $p 2$ méritent d'être confirmées par d'autres expérimentations. Les pentes $p 3$ et $p 4$ devraient varier selon la tolérance à la sécheresse des génotypes (Gate et al, 1990).

Par ailleurs, Hanks et Sorensen (1985) ont montré que l'indice de récolte du blé n'est affecté que par des contraintes hydriques d'une certaine intensité ; c'est pourquoi, en plein accord avec la figure 7 , on considère que seules les valeurs d'ETR/ETM inférieures à 0,75 auront un effet sur la variation de l'indice de récolte, quelle que soit la phase.

Enfin, d'après nos données (fig 8) et en accord avec la littérature (Steduto et al, 1986), la valeur de l'indice de récolte en deçà duquel on ne peut descendre sous l'effet d'une contrainte hydrique peut être fixé à 0,17 . Cet indice minimal permet de rendre compte des possibilités de remobilisation des réserves depuis la tige en cas de défaut d'assimilation après floraison. En conditions d'extrême aridité où un défaut complet d'épiaison peut être observé (Ouattar et Ameziane, 1989), on ne fixera pas d'indice minimal.

\section{DISCUSSION}

\section{Réponse de l'eau au blé d'hiver}

Nos résultats confirment la réponse linéaire proposée pour de nombreuses cultures (dont le blé) entre rendement ou biomasse totale et consommation hydrique (Fischer et Kohn, 1966a ; Hanks et al, 1969 ; Morizet et al, 1984 ; Howell et 
Musick, 1985). Les pentes des courbes de réponse varient néanmoins selon les auteurs : un stress de 0,50 provoque une baisse de rendement de $33 \%$ (Deumier, 1987), $48 \%$ (Drieu et Gombault, 1986), $60 \%$ (Morizet et al, 1984). En lysimètres, pour trois génotypes de blé, la chute de rendement observée est de $55 \%$ (fig 2), confirmant la synthèse de Doorenbos et Kassam (1979) pour le blé d'hiver. Les causes de variation de la pente pourraient être de deux types : le génotype et la période d'apparition du déficit hydrique. Les génotypes de blé diffèrent en effet par leur tolérance au stress hydrique (Davidson et Birch, 1978 ; Innes et Blackwell, 1981 ; Gate et al, 1990 ; Acevedo, 1991). Nous n'avons pas mis en évidence un tel effet pour les trois variétés testées. En revanche, nous avons établi des courbes de réponse qui montrent une sensibilité différentielle des phases d'élaboration du nombre de grains et de remplissage pour un même génotype.

Les valeurs d'efficience de l'eau sont comparables à celles rapportées par French et Schultz (1984), pour une large gamme de disponibilités hydriques : 4 à $19 \mathrm{~kg}$ de grain et 10 à $54 \mathrm{~kg}$ de biomasse par $\mathrm{mm}$. Nous avons montré que l'efficience était maximale dans le cas d'une sécheresse intense et continue; des valeurs de 44 à 50 $\mathrm{kg}$ de biomasse par $\mathrm{mm}$ sont également rapportées par Innes et Blackwell (1981) et Aggarwal et al (1986) pour des génotypes soumis à une sécheresse prolongée. Des effets moins prononcés sont observés par Hochman (1982), Singh et Malik (1983) et Morizet et al (1984) qui concluent plutôt à une baisse de l'efficience pour des peuplements de blé fortement stressés en eau alors qu'un déficit modéré serait plutôt favorable. Ces différences pourraient recouvrir des possibilités d'adaptation variables des cultivars de blé, en particulier de résistance stomatique (Gate et al, 1990). Si l'on soumet le blé à une contrainte progressive (SC 1991, cases profondes 1994), on permet une adaptation du blé à la contrainte et l'on augmente l'efficience. Une sécheresse brutale se traduit en revanche par une chute d'efficience. Nos observations confirment ce modèle proposé par Morizet et al (1984) pour rendre compte de l'intensité de la contrainte sur l'efficience. La valeur d'évaporation du sol nu est voisine de celle observée par ces auteurs $(50 \mathrm{~mm})$.

\section{Effet du déficit hydrique par phase}

Un fort déficit hydrique pendant le tallage est peu fréquent au champ, même en zone méridionale ; dans ces conditions, nos résultats montrent une limitation du nombre d'épillets, un arrêt précoce de l'émission des talles ou une régression rapide des talles. À la récolte, la production de matière sèche et le nombre de grains par $\mathrm{m}^{2}$ sont réduits, en conséquence d'un plus faible indice foliaire, mais l'indice de récolte est maintenu voire augmenté par un meilleur rapport puits-source qui permet de limiter la perte de rendement. De tels effets sur l'indice de récolte se dégagent des résultats de Day et al (1978), Innes et Blackwell (1981) et Talukder et al (1987). En effet, une structure d'âge plus homogène parmi la population de talles, provoquée par le déficit hydrique en TR, permet de limiter la régression des talles et augmente la quantité d'assimilats transférables par tige (Davidson et Birch, 1978). Cependant un déficit hydrique précoce, par ses conséquences négatives sur l'enracinement (Misra et al, 1969), pourrait augmenter la sensibilité du blé au manque d'eau en phase de remplissage.

Le déficit en eau courant montaison, plus fréquent, s'est traduit par une diminution du nombre de grains par $\mathrm{m}^{2}$, conséquence de l'un ou plusieurs des processus suivants : chute du nombre d'épis par $\mathrm{m}^{2}$ par suite d'une régression intense des talles et (ou) baisse du nombre de grains par épi (notamment augmentation du taux d'avortement des épillets, induction de stérilité mâle). La plus faible biomasse résulte d'une diminution de la durée de croissance et de la limitation du prélèvement d'eau. Le défaut d'alimentation azotée est induit par le dysfonctionnement racinaire et par le retard de mise à disposition de l'engrais azoté à une période de forts besoins (Sebillotte et al, 1978). Dans certains cas, la chute du nombre de grains est due à des effets directs sur les processus de fécondation, en conditions de forte sécheresse et de hautes températures autour de la floraison, qui causeraient une stérilité du pollen (Bingham, 1966).

Lorsque le déficit est prononcé dès le début de la montaison, la chute du peuplement-épi peut atteindre 35 à $40 \%$ par rapport à I'ETM (SC 1991, A95 1994) ; dans ces conditions, la réduction de biomasse et de grains par $\mathrm{m}^{2}$ est très prononcée mais le rapport puits-source est peu affecté en comparaison de I'ETM, car la réduction du nombre de puits s'accompagne d'une baisse de l'indice foliaire. Au champ, il est plus courant d'observer des réductions de l'ordre de 10 à $25 \%$ du nombre d'épis (Decau et Pujol, 1982 ; Hochman, 1982 ; Deumier, 1987), qui peuvent alors être compensées par des composantes ultérieures. Les déficits intervenant en fin 
de montaison (10-15 jours avant épiaison) ont réduit le nombre de fleurs fertiles par épillet; c'est à ce niveau que les différences variétales apparaissent déterminantes (Gate et al, 1990), aussi nos résultats doivent-ils être confrontés aux réponses de génotypes diversement tolérants à la sécheresse.

L'avortement des embryons peut s'opérer juste après fécondation, dans le cas d'une forte compétition pour les assimilats au sein de l'épi, exacerbée par la sécheresse (Steduto et al, 1986), ce qui pourrait expliquer la réduction de nombre de grains (par $\mathrm{m}^{2}$ et par épi) observée sur les traitements FSL ( $-19 \%$ en moyenne), qui subissent un déficit marqué après l'anthèse. L'indice de récolte est le plus sévèrement affecté en conséquence d'une réduction de la taille des enveloppes du grain et de la sénescence précoce des feuilles.

Le manque d'eau après floraison est le plus fréquent en conditions méridionales, combiné aux températures élevées : il entraîne une diminution du poids de 1000 grains par altération de la vitesse et (ou) de la durée de remplissage (Gallagher et al, 1976 ; Musick et Dusek, 1980 ; Mogensen et Talukder, 1987 ; Triboi, 1990) ; le défaut de remplissage du grain, cause du faible indice de récolte, est étroitement lié à l'accélération de la sénescence des feuilles après floraison et à la fermeture stomatique (Fischer et Kohn, 1966b ; Steduto et al, 1986 ; Gate et al, 1990). Nos résultats montrent un effet moins marqué en SLM qu'en FSL, la taille du grain n'étant pas affectée dans le premier cas.

\section{Compensation des effets d'un déficit hydrique au cours du cycle}

La sécheresse en fin de cycle, plus fréquente dans nos conditions, est moins préjudiciable au rendement qu'en cours de montaison, dans la mesure où le nombre de grains par $\mathrm{m}^{2}$ est la composante essentielle du rendement (Fischer, 1979 ; Johnson et Kanemasu, 1982).

À l'inverse, l'effet d'une sécheresse précoce peut être compensée (TR) en partie par les composantes s'établissant plus tardivement : cette compensation dépend du parcours d'élaboration du rendement et de processus physiologiques liés au génotype : durée de vie des feuilles, poids relatif de l'épi, taux de mobilisation des assimilats (Davidson et Birch, 1978 ; Musick et Dusek, 1980 ; Innes et Blackwell, 1981 ; Gate et al, 1990). Dans le cas d'une sécheresse modérée avant floraison et de conditions favorables lors du remplissage, l'augmentation du poids d'un grain peut résulter de la diminution du nombre de grains par un phénomène de répartition (Hooker et al, 1983 ; Deumier, 1987), comme l'indiquent les cases GF. Cependant, en terme de rendement, il n'y a pas de compensation complète d'un déficit hydrique prononcé même limité à des phases précoces (tableau VII).

En conditions de sécheresse post-floraison, Bidinger et al (1977) ont montré que $27 \%$ du poids du grain sont attribuables à la remobilisation des assimilats stockés dans la tige contre $13 \%$ en conditions de bonne alimentation hydrique (durée de fonctionnement des feuilles plus longue). Nos bilans de matière sèche conduisent à des valeurs plus élevées en présence de sécheresse post-floraison puisque la remobilisation intense observée peut contribuer à plus de $50 \%$ du poids du grain, comme l'ont également observé Gallagher et al (1976) (55-60\%) et Gate et al (1990) (37-45\%) pour différents génotypes. C'est ce qui a permis, en l'absence d'échaudage physiologique, d'assurer un remplissage satisfaisant en stress continu, alors que l'indice de récolte est resté limité par le faible nombre de grains et par la taille des réserves.

La compensation peut également se réaliser par la montée de nouveaux talles après levée de la contrainte hydrique, dans le cas d'une sécheresse avant gonflement (RG 1991, SG et SF 1994). Le décalage de maturité (15 jours à trois semaines) n'a pas permis de considérer réellement la production de ces épis en 1991 même si elle permet une compensation théorique significative. En 1994, 23 \% du rendement de la case SF étaient dus aux talles tardives. Talukder et al (1987) ont montré que le rendement des talles tardives peut représenter $39 \%$ de la production de grain, dans le cas d'un stress hydrique survenant du tallage à la mi-montaison et que la compensation n'est possible que si la levée de la contrainte est suffisamment rapide, comme nous l'avons illustré par les traitements TR et RG en 1991 ; l'indice de récolte de ces talles tardives est en revanche notablement réduit. Dans le cas de stress plus tardifs, la compensation est insuffisante (Hochman, 1982 ; Talukder et al, 1987), en dépit d'une augmentation du nombre d'épis.

Dans le cas d'un déficit hydrique avant floraison, on observe une réduction à la fois du nombre de grains et de la surface foliaire, notamment de celle des deux dernières feuilles. Ceci peut permettre de mieux ajuster le rapport sour- 
ce-puits dans le cadre d'une sécheresse après floraison, en limitant la compétition pour les assimilats (Aggarwal et Sinha, 1987) ; la réduction de la surface foliaire peut permettre des économies d'eau valorisables après l'anthèse en termes d'indice de récolte (Passioura, 1977). Ceci concerne la période pendant laquelle l'indice foliaire est inférieur à 3 et la transpiration directement liée à cet indice. Nos résultats illustrent ce fait : l'optimum d'ETR relative en post-floraison est d'environ $30 \%$ de I'ETR totale, ce qui impose de raisonner la croissance végétative en fonction des disponibilités hydriques au cours du remplissage (effet négatif d'une croissance rapide sur les cases 1994 sans irrigation ultérieure). La réduction précoce de la croissance peut être une stratégie d'adaptation aux faibles disponibilités hydriques ultérieures, par le biais de la date et la densité de semis, du génotype, des doses d'engrais (Fischer et Kohn, 1966b ; Passioura, 1977 ; Fischer, 1979 ; Nielsen et Halvorson, 1991) ; en revanche, en conditions hydriques moins sévères, il faut rechercher une forte croissance avant l'anthèse, même si l'indice de récolte peut être réduit (Entz et Fowler, 1989) ou rechercher un nombre d'épis élevé (Innes et al, 1981).

L'effet de périodes de sécheresse combinées au cours du cycle n'a pu être abordé dans ces travaux. En effet, un déficit hydrique précoce (TR) affecte en parallèle la croissance des racines et des parties aériennes, le développement des feuilles et des organes reproducteurs (mise en place du nombre de grains) ; son incidence sur le remplissage du grain en présence d'une sécheresse de post-floraison est peu prévisible (Singh et Malik, 1983). La parcours idéal de consommation de la réserve du sol et les conséquences en termes de conduite du blé pourraient être plus facilement définis par simulation.

\section{CONCLUSION}

L'indice de récolte du blé est apparu particulièrement sensible au défaut d'alimentation hydrique, alors que d'autres auteurs concluent à une relative stabilité d'IR (Hochman, 1982 ; Hanks et Sorensen, 1985). La réponse d'IR est complexe, notamment pour les stress modérés. Néanmoins, les résultats obtenus en lysimètres nous ont permis de proposer un modèle simple de réponse de l'indice de récolte par phase, en fonction de l'intensité, de la durée et de la période d'action de la contrainte hydrique, qui peut maintenant être intégré à un modèle plus complexe comme
Epic (Williams et al, 1989), les effets du déficit hydrique sur la croissance et l'indice foliaire étant déjà pris en charge par ce dernier. Cette double voie de simulation permet d'approcher le fonctionnement des relations source-puits pour différents scénarios de sécheresse et différents degrés de tolérance du blé. Elle ne doit pas masquer un certain empirisme de l'approche : ainsi, une réduction d'indice de récolte traduira, selon les phases, soit une limitation par la source ou le puits soit une limitation du transfert des assimilats de la source vers le puits.

\section{REMERCIEMENTS}

Les auteurs remercient l'ensemble des techniciens qui ont contribué à la mise en place des dispositifs, au suivi et au recueil des données, et tout spécialement $M$ Dabasse, M Hernandez, D Raffaillac et J Salvy.

\section{RÉFÉRENCES}

Acevedo E (1991) Improvement of winter cereal crops in Mediterranean environments. Use of yield, morphological and physiological traits. Colloque "Physiology-Breeding of Winter Cereals for Stressed Mediterranean Environments", Montpellier, 3-6 juillet 1989 , Les Colloques $n^{\circ} 55$, INRA, Paris, 273-305

Aggarwal PK, Sinha SK (1987) Response of droughted wheat to mid-season water application: recovery in leaf area and its effect on grain yield. Aust $J$ Plant Physiol 14, 227-237

Aggarwal PK, Singh AK, Chaturvedi GS, Sinha SK (1986) Performance of wheat and triticale in a variable soil-water environment. II. Evapotranspiration, water use efficiency, harvest index and grain yield. Field Crops Res 13, 301-315

Bidinger F, Musgrave RB, Fischer RA (1977) Contribution of stored preanthesis assimilate to grain yield in wheat and barley. Nature (London) 270, 431-433

Bingham J (1966) Varietal response in wheat to water supply in the field and male sterility caused by a period of drought in a glasshouse experiment. Ann Appl Biol 57, 365-377

Brisson N, Delecolle R (1993) Utilisation des modèles mécanistes de culture comme outils de raisonnement de la composante génétique de la résistance à la sécheresse. Colloque "Tolérance à la sécheresse des céréales en zone méditerranéenne. Diversité et amélioration variétale», Montpellier, 1517 décembre 1992, Les Colloques $n^{\circ}$ 64, INRA, Paris, 117-125 
Choudhury PN, Kumar V (1980) The sensitivity of growth and yield of dwarf wheat to water stress at three growth stages. Irrig Sci 1, 223-231

Davidson JL, Birch JW (1978) Responses of a standard Australian and a Mexican wheat to temperature and water stress. Aust J Agric Res 29, 1091-1106

Day W, Legg BJ, French BK, Johnston AE, Lawlor DW, W De C Jeffers (1978) A drought experiment using mobile shelters: the effect of drought on barley yield, water use and nutrient uptake. J Agric Sci Camb 91, 599-623

Decau J, Pujol B (1982) Irrigation d'un blé d'hiver dans le sud-ouest de la France et interaction avec l'alimentation azotée : un exemple. CR Acad Agric Fr 69, 1219-1224

Deumier JM (1987) Bilan de quelques années d'irrigation du blé. Persp Agric 114, 11-16

Donald CM, Hamblin J (1976) The biological yield and harvest index of cereals as agronomic and plant breeding criteria. Adv Agron 28, 361-405

Doorenbos J, Kassam AH (1979) Wheat. In: Yield response to water. FAO Irrigation and Drainage Paper 33, 164-170

Drieu Y, Gombault C (1986) Irriguer les blés en sols superficiels : le cas de la plaine du Forez. Persp Agric 99, 14-17

Entz MH, Fowler DB (1989) Influence of crop water environment and dry matter accumulation on grain yield of no-till winter wheat. Can J Plant Sci 69, 367375

Fischer RA (1973) The effect of water stress at various stages of development on yield processes in wheat. In: Plant response to climatic factors, Proc Uppsala Symp 1970 (RO Slatyer, ed), UNESCO, Paris, 233241

Fischer RA (1979) Growth and water limitation to dryland wheat yield in Australia: a physiological framework. J Aust Inst Agric Sci 45, 83-94

Fischer RA (1985) The role of crop simulation models in wheat agronomy. In: Wheat growth and modelling (W Day, RK Atkin, eds), Series A: life Sciences 86, Plenum Press, New York, 237-251

Fischer RA, Kohn GD (1966a) The relationship between evapotranspiration and growth in the wheat crop. Aust J Agric Res 17, 255-267

Fischer RA, Kohn GD (1966b) The relationship of grain yield to vegetative growth and post-flowering leaf area in the wheat crop under conditions of limited soil moisture. Aust J Agric Res 17, 281-295

French RJ, Schultz JE (1984) Water use efficiency of wheat in a Mediterranean-type environment. I. The relation between yield, water use and climate. Aust $J$ Agric Res 35, 743-764

Gallagher JN, Biscoe PV, Hunter B (1976) Effects of drought on grain growth. Nature (London) 264, 541542

Gate P, Bouthier A, Woznica K, Manzo ME (1990) La tolérance des variétés de blé tendre d'hiver à la sécheresse : premier résultats ITCF. Persp Agric $145,17-23$

Hanks RJ, Sorensen RB (1985) Harvest index of spring wheat as influenced by water stress. In: Wheat growth and modelling (W Day, RK Atkin, eds), NATOASI Series, Plenum Press, New York, 205-209

Hanks RJ, Gardner HR, Florian RL (1969) Plant growth-evapotranspiration relation for several crops in the Central Great Plains. Agron J 61, 30-34

Hiler EA, Clark RN (1971) Stress day index to characterize effects of water stress on crop yields. Trans ASAE 14, 757

Hochman Z (1982) Effect of water stress with phasic development on yield of wheat grown in a semi-arid environment. Field Crops Res 5, 55-67

Hooker ML, Mohiuddin SH, Kanemasu ET (1983) The effect of irrigation timing on yield and yield components of winter wheat. Can J Plant Sci 63, 815-823

Howell TA, Musick JT (1985) Relationships of dry matter of field crops to water consumption. Conf Int "Les besoins en eau des cultures", Paris, 11-14 sept 1984, INRA Éd, 247-269

Innes P, Blackwell RD (1981) The effect of drought on the water use and yield of two spring wheat genotypes. J Agric Sci Camb 96, 603-610

Innes P, Blackwell RD, Austin RB, Ford MA (1981) The effects of selection for number of ears on the yield and water economy of winter wheat. J Agric Sci Camb 97, 523-532

Johnson RC, Kanemasu ET (1982) The influence of water availability on winter wheat yields. Can $J$ Plant Sci 62, 831-838

Marty JR, Hilaire A (1979) Effets de divers précédents culturaux sur la conduite et la production du blé d'hiver : tentative de vue d'ensemble des effets liés à l'état du sol, aux reliquats d'azote et aux résidus de récolte. Agrochimica 23, 152-163

Maruejouls B (1986) Situation céréalière en MidiPyrénées : quelle stratégie pour les exploitations ? Persp Agric, N Spécial, juin 1986, 4-8

Masse J, Orsini J, Lambert $Y$ (1989) Facteurs limitant le potentiel de deux variétés de blé. Caryopse, ITCF, Mars 1989

Misra RD, Sharma KC, Wright BC, Singh VP (1969) Critical stages in irrigation and irrigation requirements of wheat variety "Lerma Rojo". Ind J Agric Sci 389, 898-906

Mogensen VO, Talukder MSV (1987) Grain yield of spring wheat in relation to water stress. 2. Growth rate of grains during drought. Cereal Res Comm 15, 247-253

Mogensen VO, Jensen HE, Abdur Rab Md (1985) Grain yield, yield components, drought sensitivity and water use efficiency of spring wheat subjected to water stress at various growth stages. Irrig Sci 6, 131-140

Morizet J, Robelin M, Baucher G (1984) Résultats de 18 années d'observations lysimétriques sous climat limagnais. II. Étude des relations entre l'eau et la production végétale. agronomie 4, 407-416 
Musick JT, Dusek DA (1980) Planting date and water deficits effects on development and yield of irrigated winter wheat. Agron J 72, 45-52

Nielsen DC, Halvorson AD (1991) Nitrogen fertility influence on water stress and yield of winter wheat. Agron J 83, 1065-1070

Nolot JM (1983) Conduite de la culture de blé d'après les résultats de l'expérimentation Sud-Ouest de 1976 à 1981. Doc ITCF, janvier 1983, $15 \mathrm{p}+$ annexes

Ouattar S, Ameziane TE (1989) Les céréales au Maroc. De la recherche à l'amélioration des techniques de production. Éditions Toubkal, Casablanca, $123 \mathrm{p}$

Passioura JB (1977) Grain yield, harvest index and water use of wheat. J Aust Inst Agric Sci 43, 117-120

Puech J, Cuadrado S, Hernandez M (1977) Influence du type textural sur l'évaporation et le drainage de différents sols en présence ou non d'un végétal. $C R$ Acad Sci Paris 284, Série D, 1045-1048

Puech J, Zehtabian GR, Hernandez M (1981) Observations sur quelques facteurs du milieu susceptibles d'influencer la production de céréales d'hiver (blé) dans les régions méridionales de l'Europe. Influence sur les composantes du rendement. Séminaire Agrimed-CEE, "Devenir de l'azote dans la fertilisation $N$ des blés d'hiver. Influence de l'alimentation hydrique", Toulouse, 146-160

Quinones H, Cabelguenne M (1990) Use of EPIC to study cropping systems II Improved simulation of the water use, growth and harvest index in corn. Agr Med 120, 241-248

Rellier JP (1981) Analyse statistique des rendements et composantes du rendement du blé tendre sur le dispositif avec rotations d'Auzeville. In: Limites de potentialités de production du blé dans différents systèmes de culture et dans différentes zones méditerranéennes. Séminaire de Bari, 30 sept2 oct, 215-227

Ritchie JT, Otter S (1985) Description and performance of CERES-Wheat yield model: a user-oriented wheat yield model. USDA-ARS Wheat Yield Project, 159-175

Sebillotte M, Boiffin J, Caneill J, Meynard JM (1978) Sécheresse et fertilisation azotée du blé d'hiver. Essai d'analyse de situations au champ par l'étude des composantes du rendement. Bull AFES 3, 197213

Singh T, Malik DS (1983) Effect of water stress at three growth stages on the yield and water-use efficiency of dwarf wheat. Irrig Sci 4, 239-245

Steduto P, Alvino A, Magliulo V, Sisto L (1986) Analysis of the physiological and reproductive responses of five wheat varieties under rainfed and irrigated conditions in Southern Italy. In: Drought resistance in plants: physiological and genetic aspects. EEC Mtg, Amalfi, 19-23 Oct 1986, 131-149

Talukder MSV, Mogensen VO, Jensen HE (1987) Grain yield of spring wheat in relation to water stress. 1. Effect of early drought on development of late tillers. Cereal Res Comm 15, 101-107

Triboi E (1990) Modèle d'élaboration du poids du grain chez le blé tendre (Triticum aestivum em Thell). agronomie 10, 191-200

Williams JR, Jones CA, Kiniry JR, Spanel DA (1989) The EPIC crop growth model. Trans ASAE 32, 497510 